\title{
On triangular norm based axiomatic extensions of the Weak Nilpotent Minimum logic
}

\author{
Carles Noguera \\ Universitat de Lleida \\ Catalonia, Spain
}

\author{
Francesc Esteva \\ Institut d'Investigació en Intel-ligència Artificial - CSIC \\ Catalonia, Spain
}

\begin{abstract}
In this paper we carry out an algebraic investigation of the Weak Nilpotent Minimum logic (WNM) and its t-norm based axiomatic extensions. We consider the algebraic counterpart of this logic, the variety of WNM-algebras (WNM) and we prove that it is locally finite, so all its subvarieties are generated by finite chains. We give criteria to compare varieties generated by finite families of WNM-chains, in particular varieties generated by standard WNM-chains, or equivalently t-norm based axiomatic extensions of WNM, and study their standard completeness properties. We also characterize the generic WNMchains, i.e. those that generate the variety $\mathbb{W N M}$, and we give finite axiomatizations for some t-norm based extensions of WNM.

Keywords: Algebraic Logic, Fuzzy logics, Left-continuous t-norms, Mathematical Fuzzy Logic, MTL-algebras, Nilpotent Minimum Logic, Non-classical logics, Residuated lattices, Substructural logics, Varieties, Weak Nilpotent Minimum Logic, WNM-algebras.
\end{abstract}

\section{Introduction}

The logic MTL (Monoidal Triangular norm based Logic) was introduced by Esteva and Godo in [10] as a generalization of Hájek's BL logic ([17]). They conjectured that it was the basic fuzzy logic, i.e. the least logic complete with respect to a semantics given by a class of leftcontinuous triangular norms (t-norms, for short) and their residua. Indeed, this was proved to be true when Jenei and Montagna showed in [20] that MTL is complete with respect to the semantics given by the class of all left-continuous t-norms and their residua.

Moreover, following [17] and [18], an algebraic semantics for MTL logic was given in [10], the variety of bounded commutative integral residuated lattices satisfying the prelinearity equation, $(x \rightarrow y) \vee(y \rightarrow x) \approx \overline{1}$. Those algebras were called MTL-algebras. In fact, this variety, that we denote $\mathbb{M T L}$, is an equivalent algebraic semantics for MTL logic, so MTL turns out to be an algebraizable logic in the sense defined by Blok and Pigozzi in [2]. As a consequence, there is a dual order isomorphism between the lattice of subvarieties of $\mathbb{M T L}$ and the lattice of axiomatic extensions of MTL. Nevertheless, the whole structure of the lattice of axiomatic extensions of MTL is very far from being known. Only some parts of the lattice have been described so far. 
Among the well studied axiomatic extensions of MTL there is the Gödel-Dummett logic $\mathrm{G}$ (see $[15,8,17])$. It can be seen as the extension of MTL obtained by adding the axiom schema of contraction, $\varphi \rightarrow \varphi \& \varphi$. Its algebraic counterpart are the G-algebras (prelinear Heyting algebras) and its latttice of subvarieties has been completely described (see e.g. [16]). Moreover, $\mathrm{G}$ is complete with respect to the semantics given by the t-norm of the minimum. The negation associated to this t-norm is the so-called Gödel negation that maps every non-zero element to zero. Following the idea of considering fuzzy logics based on tnorms related to the minimum operation, Esteva and Godo defined in [10] two new axiomatic extensions of MTL: Nilpotent Minimum logic (NM, for short) ${ }^{1}$ and Weak Nilpotent Minimum logic (WNM, for short). The first one is complete with respect to the semantics given by Fodor's nilpotent minimum t-norm (see [12]), which is a modification of the minimum t-norm by making the negation involutive; more precisely, Fodor considers the standard involutive negation $n(x)=1-x$, and keeps the value of the minimum t-norm in the region above its graph while he forces the t-norm to be 0 under the graph. Weak Nilpotent Minimum logic is a further generalization of the idea of considering t-norms related to the minimum, since it is complete with respect all t-norms defined in the same way as Fodor's t-norm but allowing the negation to be any weak negation function (in the sense of [9]). Therefore, in order to obtain these kind of t-norms that only take value 0 or the minimum, the required axiom to define WNM from MTL is $(\varphi \& \psi \rightarrow \overline{0}) \vee(\varphi \wedge \psi \rightarrow \varphi \& \psi)$. NM is the extension of WNM with the axiom schema of involution, $\neg \neg \varphi \rightarrow \varphi$. Their equivalent algebraic semantics are the varieties of NM-algebras and WNM-algebras, $\mathbb{N M}$ and $\mathbb{W} \mathbb{N M}$, respectively. The lattice of subvarieties of $\mathbb{N M}$ was completely described by the third author in [14], thus giving a complete classification of the axiomatic extensions of NM.

In this paper we aim to extend the work done for NM-algebras and G-algebras by giving a first approach to the classification of axiomatic extensions of WNM logic (i.e. a classification of the subvarieties of $\mathbb{W N M}$ ). Moreover, $\mathbb{W N M}$ can also be seen as an interesting subvariety of both 3-contractive MTL-algebras (see $[6,19,21]$ ) and the variety $\mathbb{B P}_{0}^{+\omega}$ generated by perfect MTL-algebras plus $\omega$ points (studied in [23]). After some necessary logical and algebraic ${ }^{2}$ preliminaries in Section 2, we will survey the known results for varieties of G-algebras and NM-algebras, and then in Section 3 we will describe the simple structure of WNM-chains (focusing on WNM-chains satisfying the finite partition property) and we prove that $\mathbb{W N M}$ is a locally finite variety, so, as in the case of $\mathbb{N M}$, all subvarieties are generated by finite WNMchains. In Section 3.1 we will characterize the WNM-chains that generate the whole variety $\mathbb{W N M}$ and among them we characterize the standard ones. In Section 3.2 we focus on varieties generated by standard WNM-chains (i.e. t-norm based axiomatic extensions of WNM) and study their standard completeness properties. In Section 3.3 we discuss the problem of giving axiomatizations for the axiomatic extensions of WNM, with special attention to the extensions given by standard WNM-chains. We will end with some concluding remarks. ${ }^{3}$

\footnotetext{
${ }^{1}$ According to [25], an equivalent system had been previously defined by G. J. Wang in a paper in Chinese.

${ }^{2}$ We need to assume some background in Universal Algebra. It can be found in a good reference book such as $[5]$.

${ }^{3}$ Preliminar versions of the results presented in this paper are available in $[24,21]$.
} 


\section{Preliminaries}

WNM is the logic introduced by Esteva and Godo in [10] by means of a Hilbert-style calculus in the language $\mathcal{L}=\{\&, \rightarrow, \wedge, \overline{0}\}$ of type $\langle 2,2,2,0\rangle$, where the only inference rule is Modus Ponens and the axiom schemata are the following (taking $\rightarrow$ as the least binding connective):
$(\mathrm{A} 1) \quad(\varphi \rightarrow \psi) \rightarrow((\psi \rightarrow \chi) \rightarrow(\varphi \rightarrow \chi))$
(A2) $\quad \varphi \& \psi \rightarrow \varphi$
(A3) $\quad \varphi \& \psi \rightarrow \psi \& \varphi$
(A4) $\varphi \wedge \psi \rightarrow \varphi$
(A5) $\quad \varphi \wedge \psi \rightarrow \psi \wedge \varphi$
(A6) $\quad \varphi \&(\varphi \rightarrow \psi) \rightarrow \varphi \wedge \psi$
$(\mathrm{A} 7 \mathrm{a}) \quad(\varphi \rightarrow(\psi \rightarrow \chi)) \rightarrow(\varphi \& \psi \rightarrow \chi)$
$(\mathrm{A} 7 \mathrm{~b}) \quad(\varphi \& \psi \rightarrow \chi) \rightarrow(\varphi \rightarrow(\psi \rightarrow \chi))$
$(\mathrm{A} 8) \quad((\varphi \rightarrow \psi) \rightarrow \chi) \rightarrow(((\psi \rightarrow \varphi) \rightarrow \chi) \rightarrow \chi)$
(A9) $\quad \overline{0} \rightarrow \varphi$
$(\mathrm{A} 10) \quad \neg(\varphi \& \psi) \vee(\varphi \wedge \psi \rightarrow \varphi \& \psi)$

where $\neg$ and $\vee$ are following defined connectives:

$\neg \varphi:=\varphi \rightarrow \overline{0}$;

$\varphi \vee \psi:=((\varphi \rightarrow \psi) \rightarrow \psi) \wedge((\psi \rightarrow \varphi) \rightarrow \varphi)$

Additional connectives are defined as:

$\overline{1}:=\overline{0}$;

$\varphi \leftrightarrow \psi:=(\varphi \rightarrow \psi) \wedge(\psi \rightarrow \varphi)$.

This calculus for WNM is actually the calculus for MTL extended with the axiom (A10).

We denote the set of $\mathcal{L}$-formulae built over a denumerable set $X$ of variables by $F m_{\mathcal{L}}$. Given $\Gamma \cup\{\varphi\} \subseteq F m_{\mathcal{L}}$, we write $\Gamma \vdash_{\mathrm{WNM}} \varphi$ if, and only if, $\varphi$ is provable from $\Gamma$ in the system WNM. We write $\vdash_{\text {WNM }} \varphi$ instead of $\emptyset \vdash_{\text {WNM }} \varphi$.

The Nilpotent Minimum logic (NM, for short) is the axiomatic extension of WNM obtained by adding the axiom schema of involution:

$$
\neg \neg \varphi \rightarrow \varphi .
$$

The Gödel-Dummett logic ( $\mathrm{G}$, for short) is the axiomatic extension of WNM obtained by adding the axiom schema of contraction:

$$
\varphi \rightarrow \varphi \& \varphi \text {. }
$$

A syntactical proof (analogous to the usual proof of the deduction theorem for classical logic) shows that these three logics enjoy the following global forms of deduction-detachment theorem.

Theorem 2.1. For every set of formulae $\Gamma \cup\{\varphi, \psi\} \subseteq F m_{\mathcal{L}}$ we have:

1. $\Gamma, \varphi \vdash_{\mathrm{WNM}} \psi$ if, and only if, $\Gamma \vdash_{\mathrm{WNM}} \varphi^{2} \rightarrow \psi$

2. $\Gamma, \varphi \vdash_{\mathrm{NM}} \psi$ if, and only if, $\Gamma \vdash_{\mathrm{NM}} \varphi^{2} \rightarrow \psi$

3. $\Gamma, \varphi \vdash_{\mathrm{G}} \psi$ if, and only if, $\Gamma \vdash_{\mathrm{G}} \varphi \rightarrow \psi$

where $\varphi^{2}$ is a shortcut for $\varphi \& \varphi$. 
As it is proved in [10], an algebraic semantics for WNM is given by the class of WNMalgebras.

Definition 2.2 ([10]). Let $\mathcal{A}=\left\langle A, \&^{\mathcal{A}}, \rightarrow^{\mathcal{A}}, \wedge^{\mathcal{A}}, \vee^{\mathcal{A}}, \overline{0}^{\mathcal{A}}, \overline{1}^{\mathcal{A}}\right\rangle$ be an algebra of type $\langle 2,2,2,2,0,0\rangle$. We define a unary operation by $\neg^{\mathcal{A}} a:=a \rightarrow^{\mathcal{A}} \overline{0}^{\mathcal{A}}$. Then, $\mathcal{A}$ is a WNM-algebra if, and only if, it is a bounded commutative integral residuated lattice satisfying the following equations:

$$
\begin{gathered}
(x \rightarrow y) \vee(y \rightarrow x) \approx \overline{1}, \\
\neg(x \& y) \vee(x \wedge y \rightarrow x \& y) \approx \overline{1} .
\end{gathered}
$$

An element $a \in A$ is involutive if, and only if, $\neg^{\mathcal{A}} \neg \mathcal{A} a=a$.

$\mathcal{A}$ is an NM-algebra if, and only if, all elements are involutive, i.e. $\mathcal{A}$ satifies the equation of involution: $\neg \neg x \approx x$.

An element $a \in A$ is a negation fixpoint (or just fixpoint, for short) if, and only if, $\neg^{\mathcal{A}} a=a$. In [18] Höhle proves that there exists at most one fixpoint. ${ }^{5}$

We will say that $\mathcal{A}$ is a WNM-chain (resp. NM-chain) if, and only if, the lattice order is total.

We will denote by $\mathbb{W N M}$ and $\mathbb{N M}$ the classes of WNM-algebras and NM-algebras, respectively. It can proved that both classes are varieties and, of course, $\mathbb{N M} \subseteq \mathbb{W N M}$. The algebraic counterpart of $\mathrm{G}$ is the class of prelinear Heyting algebras and it is easy to prove that they are termwise equivalent to WNM-algebras satisfying the equation of contraction: $x \& x \approx x$, which are called $G$-algebras. The variety of G-algebras is denoted by $\mathbb{G}$.

These classes of algebras allow to define a semantical consequence in the usual way. Indeed, given $\Gamma \cup\{\varphi\} \subseteq F m_{\mathcal{L}}$ and $\mathbb{K} \subseteq \mathbb{W N M}$, the expression $\Gamma \models_{\mathbb{K}} \varphi$ means that for every $\mathcal{A} \in \mathbb{K}$ and every evaluation $v$ of the formulae in $\mathcal{A}$, we have $v(\varphi)=\overline{1}^{\mathcal{A}}$ whenever $v(\psi)=\overline{1}^{\mathcal{A}}$ for every $\psi \in \Gamma$. When $\mathbb{K}=\{\mathcal{A}\}$, we write $\Gamma \models_{\mathcal{A}} \varphi$ instead of $\Gamma \models_{\{\mathcal{A}\}} \varphi$. This semantical consequence gives the first strong completeness result for the considered logics:

Theorem 2.3 ([10]). For every set of formulae $\Gamma \cup\{\varphi\} \subseteq F m_{\mathcal{L}}$, we have:

$$
\begin{aligned}
& \text { 1. } \Gamma \vdash_{\mathrm{WNM}} \varphi \text { if, and only if, } \Gamma \models_{\mathbb{W N M}} \varphi \\
& \text { 2. } \Gamma \vdash_{\mathrm{NM}} \varphi \text { if, and only if, } \Gamma \models_{\mathbb{N M}} \varphi \\
& \text { 3. } \Gamma \vdash_{\mathrm{G}} \varphi \text { if, and only if, } \Gamma \models_{\mathbb{G}} \varphi
\end{aligned}
$$

However, this result can be strenghthened by realizing that WNM is actually an algebraizable logic in the sense of Blok and Pigozzi [2] and WNM is its equivalent algebraic semantics. This implies that every axiomatic extension of WNM is also algebraizable and there is the following dual order isomorphism between the lattice of axiomatic extensions of WNM and the lattice of subvarieties of $\mathbb{W} \mathbb{N}$ :

\footnotetext{
${ }^{4}$ The superscripts in the operations will be often omitted when they are clear from the context.

${ }^{5}$ Actually, Höhle states it for the involutive algebras but the same proof gives the result for the general non-involutive case.
} 
- If $\mathrm{L}$ is the axiomatic extension of WNM obtained by adding as axiom schemata the set of formulae $\Gamma \subseteq F m_{\mathcal{L}}$, then its equivalent algebraic semantics is the subvariety $\mathbb{L} \subseteq \mathbb{W N M}$ axiomatized by the equations $\{\varphi \approx \overline{1}: \varphi \in \Gamma\}$.

- If $\mathbb{K}$ is the subvariety of $\mathbb{W} \mathbb{N M}$ axiomatized by a set of equations ${ }^{6} \Sigma \subseteq E q_{\mathcal{L}}$, then its corresponding logic is the axiomatic extension of WNM obtained by adding $\{\varphi \leftrightarrow \psi$ : $\varphi \approx \psi \in \Sigma\}$ as axiom schemata.

The strong completeness result with respect to the variety of all WNM-algebras can be refined to the class of WNM-chains by means of the following theorem:

Theorem 2.4 ([10]). Every WNM-algebra is representable as a subdirect product of WNMchains.

Corollary 2.5 ([10]). For every set of formulae $\Gamma \cup\{\varphi\} \subseteq F m_{\mathcal{L}}$, we have:

$\Gamma \vdash_{\mathrm{WNM}} \varphi$ if, and only if, $\Gamma \models_{\mathcal{A}} \varphi$ for every WNM-chain $\mathcal{A}$.

The same results are true for every axiomatic extension of WNM, in particular for NM and $\mathrm{G}$.

Finally, the completeness of WNM, NM and G with respect to chains can be further refined to the class of standard chains, i.e. chains defined over the real unit interval $[0,1]$. Recall from [10] that the operation \& in WNM-chains defined over $[0,1]$ is given by a special kind of left-continuous triangular norm. These triangular norms (t-norms, for short) are defined in the following way. If $n$ is a negation function (a function $n:[0,1] \rightarrow[0,1]$, such that $n(1)=0$, is order-reversing, and $a \leq n(n(a))$ for every $a$, as defined in [9]) and $a, b \in[0,1]$, the operation $*_{n}$ is defined as:

$$
a *_{n} b= \begin{cases}\min \{a, b\} & \text { if } a>n(b), \\ 0 & \text { otherwise. }\end{cases}
$$

This operation $*_{n}$ is a left-continuous t-norm and its residuum is given for every $a, b \in[0,1]$ by:

$$
a \rightarrow_{n} b= \begin{cases}1 & \text { if } a \leq b, \\ \max \{n(a), b\} & \text { otherwise. }\end{cases}
$$

It is straightforward that $[0,1]_{*_{n}}:=\left\langle[0,1], *_{n}, \rightarrow_{n}, \min , \max , 0,1\right\rangle$ is a WNM-chain, and all WNM-chains over $[0,1]$ are of this form. We refer to these chains as standard WNM-chains. Notice that a standard WNM-chain given by a negation function $n$ is an NM-chain if, and only if, $n$ is involutive, i.e. $n(n(a))=a$ for every $a \in[0,1]$. It follows from the study of such negation functions in [26] that there is only one standard NM-chain up to isomorphism, namely the one given by the negation $n(x)=1-x$. We will refer to it as $[0,1]_{\mathrm{NM}}$. The left-continuous t-norm corresponding to this algebra was introduced by Fodor in [12]. For G the situation is similar: there is only one standard G-algebra and it is the one given by the minimum t-norm. We will denote it by $[0,1]_{\mathrm{G}}$.

Standard algebras provide an algebraic semantics for G, NM and WNM logics, as it was proved in [8] for G, and in [9] for NM and WNM.

\footnotetext{
${ }^{6}$ Recall that given an algebraic language $\mathcal{L}$ and a set formulae in this language $F m_{\mathcal{L}}$ over some set of variables $X$, the equations are formally defined as the expressions of the form $\varphi \approx \psi$, where $\varphi, \psi \in F m_{\mathcal{L}}$. The set of all equations over $X$ is denoted as $E q_{\mathcal{L}}$.
} 
Theorem 2.6. Let $\Gamma \cup\{\varphi\} \subseteq F m_{\mathcal{L}}$ be a set of formulae. Then:

1. $\Gamma \vdash_{\mathrm{G}} \varphi$ if, and only if, $\Gamma \models_{[0,1]_{\mathrm{G}}} \varphi$.

2. $\Gamma \vdash_{\mathrm{NM}} \varphi$ if, and only if, $\Gamma \models[0,1]_{\mathrm{NM}} \varphi$.

3. $\Gamma \vdash_{\mathrm{WNM}} \varphi$ if, and only if, $\Gamma \models_{[0,1]_{*_{n}}} \varphi$ for every negation function $n$.

This kind of result is usual called a standard completeness theorem. There are several standard completeness properties that have been already considered in several papers, specially in [7] where there is a general study of such properties for fuzzy logics from which we follow the terminology and notation.

Definition 2.7. If $\mathrm{L}$ is an axiomatic extension of $\mathrm{WNM}$ and $\mathbb{K}$ is a set of $\mathrm{L}$-chains, we say that $\mathrm{L}$ has the strong $\mathbb{K}$-completeness property, $\mathrm{S} \mathbb{K} \mathrm{C}$ for short, when for every set of formulae $T \subseteq F m_{\mathcal{L}}$ and every formula $\varphi$ it holds that $T \vdash_{\mathrm{L}} \varphi$ iff $T \models_{\mathcal{A}} \varphi$ for every $\mathrm{L}$-chain $\mathcal{A} \in \mathbb{K}$. We say that $\mathrm{L}$ has the finite strong $\mathbb{K}$-completeness property, $\mathrm{FSKC}$ for short, when the equivalence holds for every finite theory $T$. We say that $\mathrm{L}$ has the $\mathbb{K}$-completeness property, $\mathbb{K C}$ for short, when the equivalence is true for $T=\emptyset$. When $\mathbb{K}$ is the class of all standard Lalgebras, we just call the property strong standard completeness (resp. finite strong standard completeness and standard completeness), and following [7] we use the notation $\mathrm{SRC}$ (resp. $\mathrm{FS} \mathcal{R} \mathrm{C}$ and $\mathcal{R C})$.

Therefore, the theorem above states that WNM and NM enjoy the strong standard completeness. Of course, the SKC implies the FSKC, and the FSKC implies the $\mathbb{K C}$. They have their equivalent algebraic property (see [7]).

Theorem 2.8. Let $\mathrm{L}$ be an axiomatic extension of $\mathrm{WNM}$, let $\mathbb{L}$ be its equivalent variety semantics and let $\mathbb{K}$ be a set of L-chains. Then:

1. L has the $\mathbb{K} C$ if, and only if, $\mathbb{L}=\mathbf{V}(\mathbb{K})$,

2. L has the $\mathrm{FSIKC}$ if, and only if, $\mathbb{L}=\mathbf{Q}(\mathbb{K})$, and

3. L has the $\mathrm{SKC}$ if, and only if, $\mathbb{L}=\mathbf{I S P}_{\sigma-f}(\mathbb{K})$.

where $\mathbf{V}$ is the operator of generated variety, $\mathbf{Q}$ is the operator of generated quasivariety and $\mathbf{P}_{\sigma-f}$ denotes the operator of reduced products over countably complete filters.

Therefore we have the following:

1. $\mathbb{W N M}=\mathbf{V}\left(\left\{[0,1]_{*_{n}}: n\right.\right.$ is a negation function $\left.\}\right)=\mathbf{Q}\left(\left\{[0,1]_{*_{n}}: n\right.\right.$ is a negation function $\})=\mathbf{I S P}_{\sigma-f}\left(\left\{[0,1]_{*_{n}}: n\right.\right.$ is a negation function $\left.\}\right)$,

2. $\mathbb{N M}=\mathbf{V}\left([0,1]_{\mathrm{NM}}\right)=\mathbf{Q}\left([0,1]_{\mathrm{NM}}\right)=\mathbf{I S P}_{\sigma-f}\left([0,1]_{\mathrm{NM}}\right)$, and

3. $\mathbb{G}=\mathbf{V}\left([0,1]_{\mathrm{G}}\right)=\mathbf{Q}\left([0,1]_{\mathrm{G}}\right)=\mathbf{I S P}_{\sigma-f}\left([0,1]_{\mathrm{G}}\right)$.

Hence, there is a single NM-chain and a single G-chain that generate (by means of these three algebraic operators) the whole variety of NM-algebras and the variety of G-algebras respectively, while in the case of WNM-algebras we have an infinite class of generators. This result will be improved in Section 3.1, where we will characterize the generic standard WNMalgebras.

Among the lattice of axiomatic extensions of WNM that we intend to study there is a subclass of particular interest: the logics associated to one concrete t-norm. 
Definition 2.9. Let $*$ be a WNM-t-norm and consider its corresponding standard WNMchain $[0,1]_{*}$. The logic associated to $*$ is the axiomatic extension of WNM corresponding to the variety $\mathbf{V}\left([0,1]_{*}\right)$ and it will be denoted by $\mathrm{L}_{*}$.

Given a WNM-t-norm $*$ it is obvious that $\mathrm{L}_{*}$ enjoys the $\mathcal{R} \mathrm{C}$ but in fact this can be said in a more precise way: $L_{*}$ enjoys the $\mathbb{K C}$ with respect to the class $\mathbb{K}=\left\{[0,1]_{*}\right\}$ (i.e. it is not necessary to consider all the standard algebras in the variety). We will call this stronger version of the $\mathcal{R C}$, canonical $\mathcal{R C}$. The canonical FSRC and the canonical $\mathrm{S} \mathcal{R} \mathrm{C}$ are defined in the analogous way. Notice that G and NM enjoy the canonical SRC.

Finally, we need to recall some more usual algebraic notions.

Definition 2.10. Let $\mathcal{A}$ be a WNM-algebra. A filter is a set $F \subseteq A$ such that:

- $\overline{1} \in F$,

- If $a \in F$ and $a \leq b$, then $b \in F$, and

- If $a, b \in F$, then $a \& b \in F$.

$F$ is proper iff $\overline{0} \notin F$.

The family of all filters of a WNM-algebra $\mathcal{A}$ is a closure system, i.e. it is a family of subsets of $A$ closed under arbitrary intersections and containing $A$. Therefore, it makes sense to speak about the notion of generated filter. We will denote by $F^{a}$ the filter generated by an element $a$.

As it happens in all bounded commutative integral residuated lattices, we have the following one-to-one correspondence between filters and congruences.

Proposition 2.11. Let $\mathcal{A}$ be a WNM-algebra. For every filter $F \subseteq A$ we define $\Theta(F):=$ $\left\{\langle a, b\rangle \in A^{2}: a \leftrightarrow b \in F\right\}$, and for every congruence $\theta$ of $\mathcal{A}$ we define $F i(\theta):=\{a \in A$ : $\langle a, 1\rangle \in \theta\}$. Then, $\Theta$ is an order isomorphism from the set of filters onto the set of congruences and $F i$ is its inverse.

By virtue of this correspondence, we will do a notational abuse by writing $\mathcal{A} / F$ instead of $\mathcal{A} / \Theta(F)$. Given an element $a \in A,[a]_{F}$ will denote the equivalence class of $a$ w.r.t. to the congruence $\Theta(F)$.

A class $\mathbb{K}$ of algebras is locally finite (LF, for short) if, and only if, for every $\mathcal{A} \in \mathbb{K}$ and for every finite set $B \subseteq A$, the subalgebra generated by $B$ is also finite. Notice that this property is inherited by the subclasses of $\mathbb{K}$.

Let $\mathcal{L}$ be an algebraic language, let $\mathcal{A}=\left\langle A,\left\{f^{\mathcal{A}}: f \in F\right\}\right\rangle$ be an algebra of type $\mathcal{L}$ and let $B \subseteq A$ be a non-empty set. The partial subalgebra $\mathcal{B}$ of $\mathcal{A}$ with domain $B$ is the partial algebra $\left\langle B,\left\{f^{\mathcal{B}}: f \in F\right\}\right\rangle$, where for every $f \in F n$-ary, and every $b_{1}, \ldots, b_{n} \in B$,

$$
f^{\mathcal{B}}\left(b_{1}, \ldots, b_{n}\right)= \begin{cases}f^{\mathcal{A}}\left(b_{1}, \ldots, b_{n}\right) & \text { if } f^{\mathcal{A}}\left(b_{1}, \ldots, b_{n}\right) \in B, \\ \text { undefined } & \text { otherwise. }\end{cases}
$$

We denote it by $\mathcal{B} \subseteq p$ A.

Given two algebras $\mathcal{A}$ and $\mathcal{B}$ of the same language we say that $\mathcal{A}$ is partially embeddable into $\mathcal{B}$ when every finite partial subalgebra of $\mathcal{A}$ is embeddable into $\mathcal{B}$. Generalizing this notion to classes of algebras, we say that a class $\mathbb{K}$ of algebras is partially embeddable into a 
class $\mathbb{M}$ if every finite partial subalgebra of a member of $\mathbb{K}$ is embeddable into a member of M.

If the language is finite, this turns out to be equivalent to say that $\mathbb{K}$ belongs to the universal class generated by $\mathbb{M}$ (see for instance [16]). That is, by recalling tos' theorem (see [5]) of characterization of universal classes, we have the following equivalence.

Proposition 2.12 ([16]). Let $\mathbb{K}$ and $\mathbb{M}$ be classes of algebras of the same finite language. Then the following conditions are equivalent:

- $\mathbb{K}$ is partially embeddable into $\mathbb{M}$

- $\mathbb{K} \subseteq \mathbf{I S P}_{U}(\mathbb{M})$

Given a class $\mathbb{K}$ of algebras, $\mathbb{K}_{\text {fin }}$ will denote the class of its finite members.

A class $\mathbb{K}$ of algebras has the finite embeddability property (FEP, for short) if, and only if, it is partially embeddable into $\mathbb{K}_{\text {fin }}$.

A class $\mathbb{K}$ of algebras of the same type has the strong finite model property (SFMP, for short) if, and only if, every quasiequation that fails to hold in $\mathbb{K}$ can be refuted in some member of $\mathbb{K}_{\text {fin }}$.

A class $\mathbb{K}$ of algebras of the same type has the finite model property (FMP, for short) if, and only if, every equation that fails to hold in $\mathbb{K}$ can be refuted in some member of $\mathbb{K}_{\text {fin }}$.

It is clear that a variety has the FMP if, and only if, it is generated by its finite members and a quasivariety has the SFMP if, and only if, it is generated (as a quasivariety) by its finite members.

Theorem $2.13([4])$. Let $\mathcal{L}$ be a finite algebraic language and let $\mathbb{K}$ be a class of algebras of type $\mathcal{L}$ closed under finite products. Then, $\mathbb{K}$ has the FEP if, and only if, $\mathbb{K}$ has the SFMP.

Moreover, it is clear that for every class of algebras $\mathbb{K}$, we have:

- If $\mathbb{K}$ is locally finite, then it has the FEP.

- If $\mathbb{K}$ has the FEP, then it has the SFMP.

- If $\mathbb{K}$ has the SFMP, then it has the FMP.

Theorem 2.14 ([1]). Let $\mathcal{L}$ be a finite algebraic language and let $\mathbb{K}$ be a variety of algebras of type $\mathcal{L}$ enjoying the EDPC (equationally definable principal congruences property). Then, the following are equivalent:

- $\mathbb{K}$ has the FEP,

- $\mathbb{K}$ has the SFMP,

- $\mathbb{K}$ has the FMP.

Since for every algebraizable logic whose equivalent algebraic semantics is a variety, the EDPC of the equivalent algebraic semantics is equivalent to the deduction-detachment theorem of the logic (see [3]) and these logics enjoy it (as stated in Theorem 2.1), we can conclude that WNM and all its axiomatic extensions fall under the conditions of the last theorem. 


\subsection{Varieties of G-algebras and NM-algebras}

The structure of $\mathrm{G}$-chains and the lattice of subvarieties of $\mathbb{G}$ are well known (see for instance [16]). Indeed, if $\mathcal{A}=\left\langle A, \&^{\mathcal{A}}, \rightarrow^{\mathcal{A}}, \wedge^{\mathcal{A}}, \vee^{\mathcal{A}}, \overline{0}^{\mathcal{A}}, \overline{1}^{\mathcal{A}}\right\rangle$ is a G-chain, then for every $a, b \in A$ :

$$
\begin{gathered}
a \&^{\mathcal{A}} b=a \wedge^{\mathcal{A}} b \\
a \rightarrow^{\mathcal{A}} b= \begin{cases}\overline{1}^{\mathcal{A}} & \text { if } a \leq b, \\
b & \text { otherwise. }\end{cases}
\end{gathered}
$$

We can consider the following canonical finite G-chains. For every $n \geq 1$ the canonical G-chain of $n$ elements is defined as $\mathcal{G}_{n}:=\langle\{0, \ldots, n-1\}, \&, \rightarrow, \wedge, \vee, 0, n-1\rangle$, where for every $a, b \in\{0, \ldots, n-1\}, a \& b=a \wedge b=\min \{a, b\}, a \vee b=\max \{a, b\}$ and

$$
a \rightarrow b= \begin{cases}n-1 & \text { if } a \leq b, \\ b & \text { otherwise }\end{cases}
$$

Every finite G-chain with exactly $n$ elements is isomorphic to $\mathcal{G}_{n}$.

It is straigthforward to check that $\mathbb{G}$ is a locally finite variety, thus all varieties of $\mathrm{G}$ algebras are generated by finite G-chains. Morever, since $\mathcal{G}_{n} \subseteq \mathcal{G}_{n+1}$ for every $n \geq 1$, then the varieties generated by a finite family of finite G-chains form the following chain: $\mathbf{V}\left(\mathcal{G}_{1}\right) \subseteq$ $\mathbf{V}\left(\mathcal{G}_{2}\right) \subseteq \mathbf{V}\left(\mathcal{G}_{3}\right) \subseteq \ldots \subseteq \mathbf{V}\left(\mathcal{G}_{n}\right) \subseteq \mathbf{V}\left(\mathcal{G}_{n+1}\right) \subseteq \ldots$ Finally, every infinite family of finite G-chains generates the whole variety $\mathbb{G}$, so there are no more subvarieties.

It is clear that $\mathbf{V}\left(\mathcal{G}_{1}\right)$ is the trivial variety and $\mathbf{V}\left(\mathcal{G}_{2}\right)$ is (termwise equivalent to) the variety of Boolean algebras. For every $n \geq 3$, an axiomatization (relative to $\mathbb{G}$ ) for $\mathbf{V}\left(\mathcal{G}_{n}\right)$ is given by the equation: $\bigvee_{i<n}\left(x_{i} \rightarrow x_{i+1}\right) \approx \overline{1}$. This implies that the inclusions in the chain of varieties are strict.

As regards to $\mathbb{N M}$, its lattice of subvarieties has been completely described in [14]. We will briefly present this description.

The structure of finite NM-chains is very simple. In fact, for every $n \geq 1$ there is exactly one, up to isomorphism, NM-chain with $n$ elements. Therefore, we can also define canonical finite NM-chains.

For every $n \geq 1$ the canonical NM-chain of $2 n$ elements is defined as $\mathcal{N}_{2 n}:=\langle\{-n,-(n-$ 1) $, \ldots,-1,1, \ldots, n-1, n\}, \&, \rightarrow, \wedge, \vee,-n, n\rangle$ and the canonical NM-chain of $2 n+1$ elements is defined as $\mathcal{N}_{2 n+1}:=\langle\{-n,-(n-1), \ldots,-1,0,1, \ldots, n-1, n\}, \&, \rightarrow, \wedge, \vee,-n, n\rangle$, where:

$$
\begin{aligned}
a \& b & := \begin{cases}\min \{a, b\} & \text { if } a>-b, \\
-n & \text { otherwise. }\end{cases} \\
a \rightarrow b & := \begin{cases}n & \text { if } a \leq b, \\
\max \{-a, b\} & \text { otherwise. }\end{cases}
\end{aligned}
$$

$a \wedge b:=\min \{a, b\}$ and $a \vee b:=\max \{a, b\}$.

Recall also the definition of the unique (up to isomorphism) standard NM-chain, $[0,1]_{\mathrm{NM}}$.

Given an NM-chain $\mathcal{C}$ with fixpoint, we denote by $\mathcal{C}^{-}$the subalgebra obtained by erasing the fixpoint. With this notation, it is clear that $\mathcal{N}_{2 n}=\mathcal{N}_{2 n+1}^{-}$for every $n \geq 1$.

Theorem 2.15 ([14]). A variety of NM-algebras is a proper subvariety of $\mathbb{N M}$ if, and only if, it does not contain $\mathcal{N}_{n}$ for some $n \geq 1$. 
Corollary 2.16 ([14]). If $\mathcal{A}$ is an infinite NM-chain with fixpoint, then $\mathbf{V}(\mathcal{A})=\mathbb{N M}$.

Theorem 2.17 ([14]). NM is locally finite.

This fact, as already discussed, implies the FMP and the decidability of NM. Thus, in particular, we have that every variety of NM-chains is generated by its finite chains. It leads to the following classification and axiomatization of the subvarieties of $\mathbb{N M}$ :

Theorem 2.18 ([14]). Consider the terms $B p(x)=\left(\neg(\neg x)^{2}\right)^{2} \leftrightarrow \neg\left(\neg x^{2}\right)^{2}$ and $S_{n}\left(x_{0}, \ldots, x_{n}\right)=$ $\bigwedge_{i<n}\left(\left(x_{i} \rightarrow x_{i+1}\right) \rightarrow x_{i+1}\right) \rightarrow \bigvee_{i<n+1} x_{i}$. Every proper subvariety of $\mathbb{N M}$ is of one of the following types:

1. $\mathbf{V}\left([0,1]_{\mathrm{NM}}^{-}\right)=\mathbf{V}\left(\left\{\mathcal{N}_{2 n}: n \geq 1\right\}\right)$ and it is axiomatized by $B p(x) \approx \overline{1}$, or

2. $\mathbf{V}\left(\mathcal{N}_{2 n+1}\right)$ for some $n \in \omega$ and it is axiomatized by $S_{n}\left(x_{0}, \ldots, x_{n}\right) \approx \overline{1}$, or

3. $\mathbf{V}\left(\mathcal{N}_{2 n}\right)$ for some $n \in \omega$ and it is axiomatized by $S_{n}\left(x_{0}, \ldots, x_{n}\right) \approx \overline{1}$ and $B p(x) \approx \overline{1}$, or

4. $\mathbf{V}\left([0,1]_{\mathrm{NM}}^{-}, \mathcal{N}_{2 n+1}\right)$ for some $n \in \omega$ and it is axiomatized by $B p(x) \vee S_{n}\left(x_{0}, \ldots, x_{n}\right) \approx \overline{1}$, or

5. $\mathbf{V}\left(\mathcal{N}_{2 n}, \mathcal{N}_{2 m+1}\right)$, for some $m, n \in \omega$ such that $m<n$ and it is axiomatized by $(B p(x) \wedge$ $\left.S_{n}\left(x_{0}, \ldots, x_{n}\right)\right) \vee S_{m}\left(x_{0}, \ldots, x_{m}\right) \approx \overline{1}$.

Therefore, the paper [14] gives a complete description of all axiomatic extensions of NM. The lattice of all these logics is depicted in Figure 1, where $\mathrm{NM}^{-}$denotes the logic corresponding to $\mathbf{V}\left([0,1]_{\mathrm{NM}}^{-}\right)$, NM $n$ denotes the logic corresponding to $\mathbf{V}\left(\mathcal{N}_{n}\right)$, NMnm denotes the logic corresponding to $\mathbf{V}\left(\mathcal{N}_{n}, \mathcal{N}_{m}\right)$, and $\mathrm{NM} n, \mathrm{NM}^{-}$denotes the logic corresponding to $\mathbf{V}\left(\mathcal{N}_{n},[0,1]_{\mathrm{NM}}^{-}\right)$.

\section{Main results}

\subsection{General facts about WNM-chains}

Starting from the results of the last section our aim now is to study the whole variety of WNM-algebras and its subvarieties. First we need to study the WNM-chains, since they generate all the subvarieties of $\mathbb{W} \mathbb{N M}$.

The operations in WNM-chains are quite simple as the following lemma states:

Lemma 3.1. Let $\mathcal{A}=\langle A, \&, \rightarrow, \wedge, \vee, \overline{0}, \overline{1}\rangle$ be a WNM-chain. Then for every $a, b \in A$ :

$$
\begin{gathered}
a \& b= \begin{cases}a \wedge b & \text { if } a>\neg b, \\
\overline{0} & \text { otherwise. }\end{cases} \\
a \rightarrow b= \begin{cases}\overline{1} & \text { if } a \leq b, \\
\neg a \vee b & \text { otherwise. }\end{cases}
\end{gathered}
$$

Notice that the previous lemma generalizes the structure of standard WNM-chains presented in the preliminaries. It turns out, that WNM-chains depend just on the order and the negation operation, thus we need to recall some properties of such operations.

Lemma 3.2. Let $\mathcal{A}$ be a WNM-chain. Then for every $a \in A$ : 


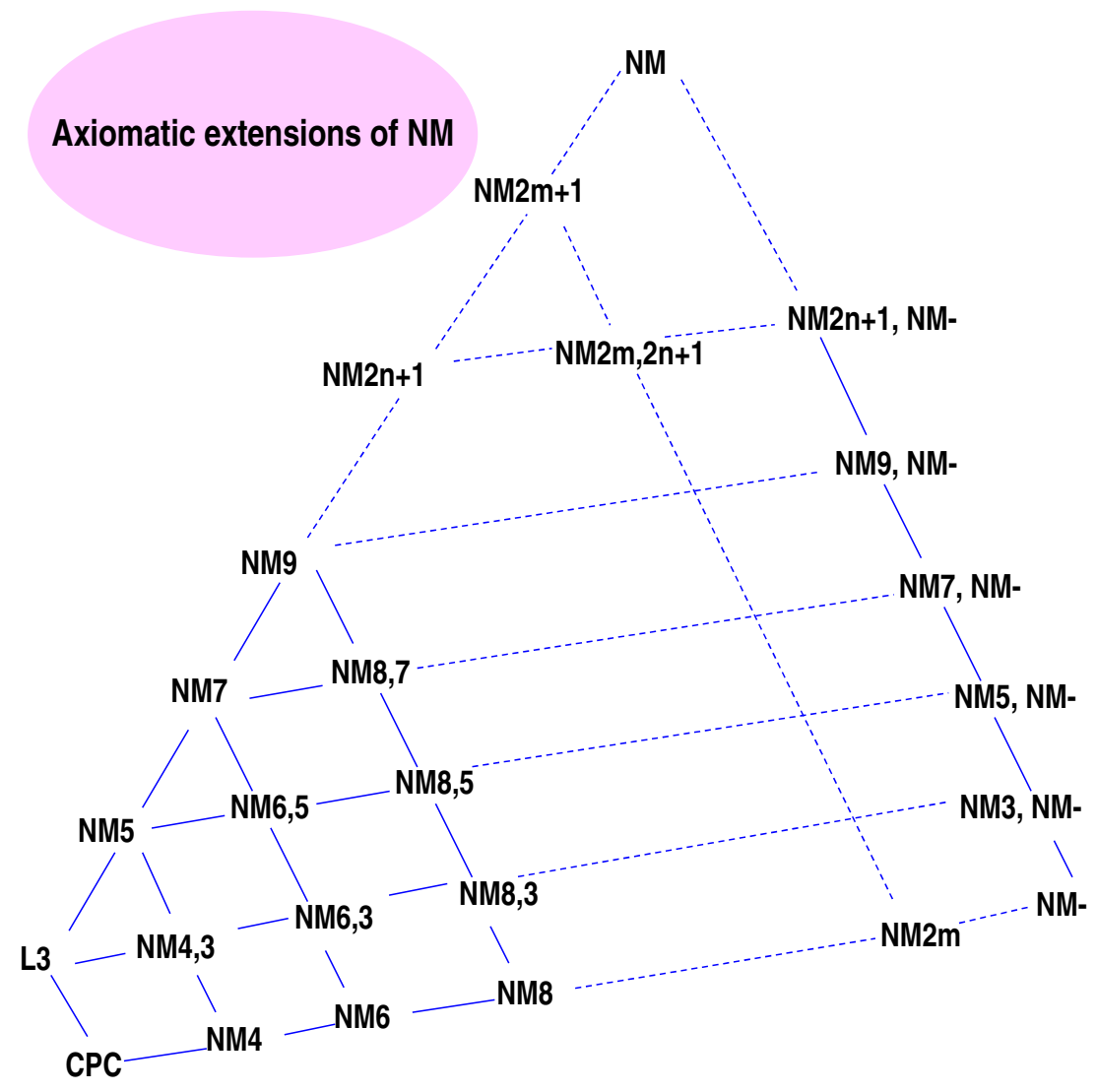

Figure 1: Lattice of axiomatic extensions of NM.

(i) $\neg a=\neg \neg \neg a$,

(ii) $a \leq \neg \neg a$,

(iii) $a=\neg \neg a$ if, and only if, there is $b \in A$ such that $a=\neg b$, and

(iv) $\neg \neg a=\min \{b \in A: a \leq b$ and $b=\neg \neg b\}$.

The negation operation determines two kinds of elements in a chain: the positive and the negative, which are defined as follows.

Definition 3.3. Given a WNM-algebra $\mathcal{A}$, the sets of positive and negative elements are respectively defined as:

$$
\begin{aligned}
& A_{+}:=\{a \in A: a>\neg a\} \\
& A_{-}:=\{a \in A: a \leq \neg a\}
\end{aligned}
$$

Consider the terms $p(x):=x \vee \neg x$ and $n(x):=x \wedge \neg x$. The next proposition gives a useful way to describe these sets:

Proposition 3.4. Let $\mathcal{A}$ be a WNM-algebra. Then: 
- $A_{+}=\{p(a): a \in A, \neg a \neq \neg \neg a\}$.

- $A_{-}=\{n(a): a \in A\}$.

Next we will prove that the involutive elements of a WNM-chain form an NM-algebra.

Definition 3.5. Let $\mathcal{A}$ be a WNM-chain. $N(\mathcal{A})$ will denote the set of involutive elements of $\mathcal{A}$, i.e. $N(\mathcal{A})=\{\neg a: a \in A\}$.

Proposition 3.6. Let $\mathcal{A}$ be a WNM-chain. Then $N(\mathcal{A})$ is the universe of the maximum NM-subalgebra of $\mathcal{A}$. We denote it by $\mathcal{N}(\mathcal{A})$.

Proof. We must prove that $N(\mathcal{A})$ is closed under all operations. Obviously, $\overline{0}=\neg \overline{1} \in N(\mathcal{A})$ and $\overline{1}=\neg \overline{0} \in N(\mathcal{A})$. Take $\neg a, \neg b \in N(\mathcal{A})$. Since $\mathcal{A}$ is linearly ordered, $\neg a \wedge \neg b, \neg a \vee \neg b \in$ $N(\mathcal{A})$, hence $\neg a \& \neg b \in N(\mathcal{A})$. Finally, if $\neg a \leq \neg b$, then $\neg a \rightarrow \neg b=\overline{1} \in N(\mathcal{A})$; otherwise $\neg a \rightarrow \neg b=\neg \neg a \vee \neg b \in N(\mathcal{A})$.

Proposition 3.7. Let $\mathbb{K} \subseteq \mathbb{W N M}$ be a variety. Then, $\mathbb{K} \cap \mathbb{N M}=\mathbf{V}(\{\mathcal{N}(\mathcal{A}): \mathcal{A}$ chain of $\mathbb{K}\})$.

Proof. The inclusion from right to left is clear, since for every chain of $\mathbb{K}, \mathcal{A}$, we have that $\mathcal{N}(\mathcal{A})$ is an NM-chain and it is a subalgebra of an algebra in $\mathbb{K}$, so $\mathcal{N}(\mathcal{A}) \in \mathbb{K} \cap \mathbb{N M}$. Conversely, if $\mathcal{C}$ is a chain of $\mathbb{K} \cap \mathbb{N M}$, then $\mathcal{C}=\mathcal{N}(\mathcal{C}) \in\{\mathcal{N}(\mathcal{A}): \mathcal{A}$ chain of $\mathbb{K}\}$, and by the subdirect representation theorem, we obtain the inclusion.

To deal with the non-involutive elements we will use the following definition.

Definition 3.8. Let $\mathcal{A}$ be a WNM-chain and let $a \in A$ be an involutive element. We define $I_{a}^{\mathcal{A}}:=\{b \in A: \neg b=\neg a\}$ and we call it the interval associated to a, where the negation function is constant with value $\neg$. We say that a has a trivial associated interval when $I_{a}^{\mathcal{A}}=\{a\}$. When $\mathcal{A}$ is a standard WNM-chain given by a t-norm $*$, we will sometimes write $I_{a}^{*}$ instead of $I_{a}^{\mathcal{A}}$. We will write just $I_{a}$ when the algebra is clear from the context.

Now we can define the finite partition property for WNM-chains.

Definition 3.9. Let $\mathcal{A}$ be a WNM-chain. We say that $\mathcal{A}$ enjoys the finite partition property (FPP, for short) iff $\neg^{\mathcal{A}}$ is constant in a finite number of intervals, i.e. the set $\left\{a \in A: I_{a} \neq\right.$ $\{a\}\}$ is finite. Let $I_{a_{1}}, \ldots, I_{a_{n}}$ be these intervals. In such a case we define the associated finite partition $P$ in the following way:

- $I_{a_{1}}, \ldots, I_{a_{n}} \in P$.

- Consider the set $X=A \backslash\left(I_{a_{1}} \cup \ldots \cup I_{a_{n}}\right)$. It is clear that $X \subseteq N(\mathcal{A})$. For every connected component $Y$ of $X \cap A_{-}$, consider the elements $\neg a_{i_{1}}<\ldots<\neg a_{i_{k}} \in Y$ (where $\left.\left\{i_{1}, \ldots, i_{k}\right\} \subseteq\{1, \ldots, n\}\right)$, and add the following intervals to $P$ : $Y \cap\left[0, \neg a_{i_{1}}\right],\left(\neg a_{i_{1}}, \neg a_{i_{2}}\right], \ldots,\left(\neg a_{i_{k-1}}, \neg a_{i_{k}}\right], Y \cap\left(\neg a_{i_{k}}, 1\right]$. If there are no elements of the form $\neg a_{i}$ in $Y$, we add $Y$ to $P$. We do the same for every connected component $Y$ of $X \cap A_{+}$.

Notice that this partition yields two kinds of intervals: those where the negation takes a constant value, and those where all elements are involutive. As a matter of nomenclature, we call them constant intervals and involutive intervals, respectively. Figure 2 shows an example of a WNM-t-norm with negation fixpoint, $a_{3}$, and satisfying the FPP where the constant 


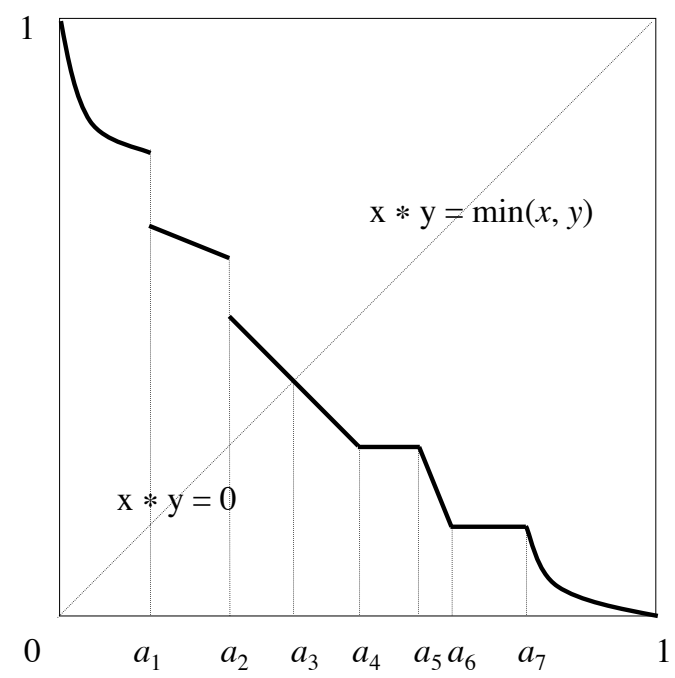

Figure 2: An example of WNM-t-norm satisfying the FPP.

intervals are $\left[a_{4}, a_{5}\right]$ and $\left[a_{6}, a_{7}\right]$, while the involutive intervals are $\left[0, a_{1}\right],\left(a_{1}, a_{2}\right],\left(a_{2}, a_{3}\right]$, $\left(a_{3}, a_{4}\right),\left(a_{5}, a_{6}\right)$ and $\left(a_{7}, 1\right]$.

Figure 3 shows three families of WNM-t-norms enjoying the FPP parametrized with a real number $c: c \in[0,1)$ for $\otimes_{c}, c \in[1 / 2,1)$ for $\star_{c}$ and $c \in[1 / 2,1]$ for $\odot_{c}$. Notice that $\otimes_{0}=\odot_{1}=\min$ and $\star_{1 / 2}=\odot_{1 / 2}$ is the nilpotent minimum t-norm. Actually, these families are the only WNM-t-norms having a finite partition of at most three intervals.

To refer to the class of WNM-t-norms and those satisfying the FPP we will use from now on the following notations:

$\mathbf{W N M}=\{*$ is a weak nilpotent minimum t-norm $\}$

$\mathbf{W N M - f i n}=\left\{* \in \mathbf{W N M} \mid[0,1]_{*}\right.$ enjoys the FPP $\}$

Next we prove that the variety of WNM-algebras is locally finite.

Lemma 3.10. Let $\mathcal{A}$ be a WNM-chain. Then, every finite subset of $A$ generates a finite WNM-chain.

Proof. Take a finite subset $B=\left\{b_{0}, \ldots, b_{n}\right\} \subseteq A$. Due to Lemma 3.1 and (i) of Lemma 3.2 it is obvious that the universe of the subalgebra generated by $B$ is $\left\{\overline{0}, \overline{1}, b_{0}, \ldots, b_{n}, \neg b_{0}, \ldots, \neg b_{n}, \neg \neg b_{0}, \ldots, \neg \neg b_{n}\right\}$, so it is finite.

Proposition 3.11. $\mathbb{W N M}$ is a locally finite variety.

Proof. Let $\mathcal{A}$ be a WNM-algebra and take a finite set $B \subseteq A$. Suppose that $B=\left\{b_{0}, \ldots, b_{n}\right\}$. We must prove that $\langle B\rangle_{\mathcal{A}}$ is also finite. If $\mathcal{A}$ is a chain, we apply the previous lemma. Suppose that $\mathcal{A}$ is not a chain. Then, due to the theorem of representation in subdirect products of chains, we have an embedding $\alpha: \mathcal{A} \hookrightarrow \prod_{i \in I} \mathcal{A}_{i}$, where for every $i \in I, \mathcal{A}_{i}$ is a WNM-chain. 


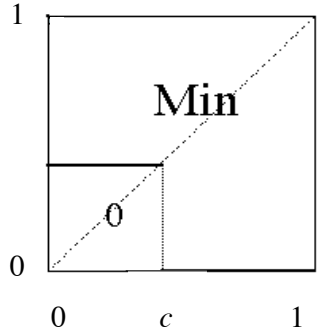

$\otimes c$

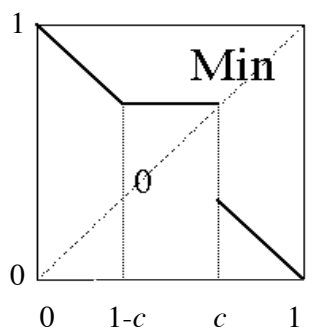

$\star c$

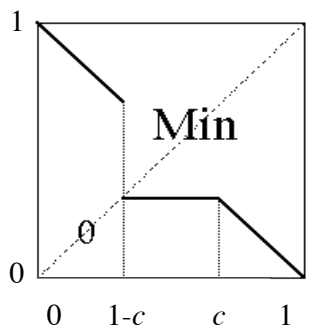

$\odot c$

Figure 3: Three parametric families of WNM-t-norms enjoying the FPP.

Consider the images of the elements of $B, \alpha\left(b_{j}\right)=\left(a_{i}^{j}\right)_{i \in I}$, for every $j \in\{1, \ldots, n\}$. We have seen that for every $i \in I,\left\{a_{i}^{1}, \ldots, a_{i}^{n}\right\}$ generates a finite chain $\mathcal{C}_{i} \subseteq \mathcal{A}_{i}$ whose universe is $\left\{\overline{0}^{\mathcal{A}_{i}}, \overline{1}^{\mathcal{A}_{i}}, a_{i}^{1}, \ldots, a_{i}^{n}, \neg a_{i}^{1}, \ldots, \neg a_{i}^{n}, \neg \neg a_{i}^{1}, \ldots, \neg \neg a_{i}^{n}\right\}$. Notice that there is only a finite number of such chains up to isomorphism, say $\left\{\mathcal{C}_{0}, \ldots, \mathcal{C}_{n-1}\right\}$, and $\langle B\rangle_{\mathcal{A}} \in \mathbf{V}\left(\left\{\mathcal{C}_{i}: i<n\right\}\right)$. Therefore, using that every variety generated by a finite number of finite algebras is locally finite ([5], Theorem 10.16), we obtain that $\langle B\rangle_{\mathcal{A}}$ is finite.

We have the following easy consequences:

- $\mathbb{W} \mathbb{N}$ has the FEP.

- $\mathbb{W} N M$ has the FMP.

- $\mathbb{W N M}=\mathbf{V}\left(\mathbb{W N M}_{f i n}\right)=\mathbf{Q}\left(\mathbb{W N M}_{f i n}\right)$.

- Every subvariety of $\mathbb{W N M}$ is generated (as a variety and as a quasivariety) by its finite chains.

- The quasiequational theory of every finitely axiomatizable subvariety of $\mathbb{W} \mathbb{N M}$ is decidable.

- WNM and all its finitely axiomatizable extensions are decidable. ${ }^{7}$

Lemma 3.12. Let $\mathcal{A}$ be a WNM-chain, let $F$ be a filter and consider the quotient algebra $\mathcal{A} / F$. Then:

- $\left[\overline{1}^{\mathcal{A}}\right]_{F}=F$

- $\left[\overline{0}^{\mathcal{A}}\right]_{F}=\{a \in A: \neg a \in F\}$

- For every $a, b \in A \backslash\left(\left[\overline{1}^{\mathcal{A}}\right]_{F} \cup\left[\overline{0}^{\mathcal{A}}\right]_{F}\right)$ such that $a \neq b$, we have $[a]_{F} \neq[b]_{F}$.

\footnotetext{
${ }^{7}$ The decidability of WNM and some of its extensions (and expansions with truth-constants) was already proved in [11] with a different reasoning which, in fact, proved that for these logics the tautology and the consequence problems are coNP-complete while the satisfaction problem is NP-complete.
} 
Proof. The first statement is trivial. As for the second, take an arbitrary $a \in A$. Then, $a \in\left[\overline{0}^{\mathcal{A}}\right]_{F}$ iff $a \rightarrow \overline{0}^{\mathcal{A}} \in F$ iff $\neg a \in F$. Now consider a pair of different elements $a, b \in$ $A \backslash\left(\left[\overline{1}^{\mathcal{A}}\right]_{F} \cup\left[\overline{0}^{\mathcal{A}}\right]_{F}\right)$. Suppose, for instance, that $a>b$. Then, $a \rightarrow b=\neg a \vee b \notin F$, hence $[a]_{F} \neq[b]_{F}$.

Roughly speaking the last lemma describes homomorphic images (i.e. quotients) of WNMchains as the result of identifying all the elements of a filter in the top of the resulting chain, identifying the elements whose negation is in the filter in the bottom element, and leaving the rest of the chain as it was. Figure 4 shows the quotient of a standard WNM-chain.

Lemma 3.13. Let $\mathcal{A}$ and $\mathcal{B}$ be WNM-chains and let $f: \mathcal{A} \rightarrow \mathcal{B}$ a surjective homomorphism. Then:

(i) If $I_{\overline{1}^{\mathcal{B}}}=\left\{\overline{1}^{\mathcal{B}}\right\}$, then $\mathcal{B}$ is embeddable into $\mathcal{A}$.

(ii) If $I_{\overline{1}^{\mathcal{B}}} \neq\left\{\overline{1}^{\mathcal{B}}\right\}$, then there is $a \in N(\mathcal{A}) \cap A_{+}$such that $I_{a} \neq\{a\}$ and $\mathcal{B}$ is embeddable into $\mathcal{A} / F^{a}$.

Proof. By the Homomorphism Theorem we know that $\mathcal{A} / \operatorname{Kerf} \cong \mathcal{B}$; thus, after the previous lemma, we can assume that the universe of $\mathcal{B}$ is $\left(A \backslash\left(\left[\overline{1}^{\mathcal{A}}\right]_{\text {Kerf }} \cup\left[\overline{0}^{\mathcal{A}}\right]_{\text {Kerf }}\right)\right) \cup\left\{\overline{0}^{\mathcal{A}}, \overline{1}^{\mathcal{A}}\right\}$. (i) is obvious. Assume that $I_{\overline{1}^{\mathcal{B}}} \neq\left\{\overline{1}^{\mathcal{B}}\right\}$. Take $c \in I_{\overline{1}^{\mathcal{B}}} \backslash\left\{\overline{1}^{\mathcal{B}}\right\}$, then it is clear that $\mathcal{B}$ is embeddable into $\mathcal{A} / F\urcorner \neg c$.

Corollary 3.14. Let $\mathcal{A}$ be a WNM-chain. Then, $\mathbf{H}(\mathcal{A})=\mathbf{I S}(\mathcal{A}) \cup \mathbf{I S}\left(\left\{\mathcal{A} / F^{a}: a \in N(\mathcal{A}) \cap A_{+}\right.\right.$ and $\left.\left.I_{a} \neq\{a\}\right\}\right)$. Moreover, if there exists the maximum positive involutive element a with $I_{a} \neq\{a\}$ and such that for any other $b \in N(\mathcal{A}) \cap A_{+}, I_{b}$ is order-embeddable into $I_{a}$, then $\mathbf{H}(\mathcal{A})=\mathbf{I S}(\mathcal{A}) \cup \mathbf{I S}\left(\mathcal{A} / F^{a}\right)$.

Proof. All the algebras of $\mathbf{H}(\mathcal{A})$ are a homomorphic image of $\mathcal{A}$ and thus, by the previous lemma, they are embeddable into $\mathcal{A}$ or into $\mathcal{A} / F^{a}$ for some $a \in N(\mathcal{A}) \cap A_{+}$. Assume now that there exists the maximum positive involutive element $a$ with $I_{a} \neq\{a\}$ and such that for any other $b \in N(\mathcal{A}) \cap A_{+}, I_{b}$ is order-embeddable into $I_{a}$. According to the description of quotients of WNM-chains given in Lemma 3.12, for every $b \in N(\mathcal{A}) \cap A_{+}$such that $b<a$, the assumption implies that $\mathcal{A} / F^{b}$ is embeddable into $\mathcal{A} / F^{a}$, and hence $\mathbf{I S}\left(\mathcal{A} / F^{b}\right) \subseteq \mathbf{I S}\left(\mathcal{A} / F^{a}\right)$.

Notice that for every standard WNM-chain $[0,1]_{*}$ whose t-norm is in WNM-fin, there is a maximum positive involutive element $a$ such that $I_{a} \neq\{a\}$ (possibly $a=1$ ) and, since all the constant intervals have the same order-type, we have $\mathbf{H}\left([0,1]_{*}\right)=\mathbf{I S}\left([0,1]_{*}\right) \cup \mathbf{I S}\left([0,1]_{*} / F^{a}\right)$. Actually, the algebra $[0,1]_{*} / F^{a}$ can also be seen as a standard WNM-chain since it is clearly isomorphic to a chain over $[0,1]$. The reader can see an example of such situation in Figure 4.

Lemma 3.15. Let $\mathbb{K}$ be a class of WNM-chains closed under subalgebras. We have: $\mathbf{H}(\mathbb{K})_{\text {fin }}=$ $\mathbf{H}\left(\mathbb{K}_{\text {fin }}\right)$.

Proof. One inclusion is trivial. As for the other one, take $\mathcal{A} \in \mathbf{H}(\mathbb{K})_{\text {fin }}$, then $\mathcal{A}$ is a finite chain and it is isomorphic to $\mathcal{B} / F$ for some $\mathcal{B} \in \mathbb{K}$ and some filter $F$ of $\mathcal{B}$. Define $\bar{F}=\{a \in$ $A \mid \neg a \in F\}$. The subalgebra of $\mathcal{B}$ generated by $B \backslash(F \cup \bar{F})$ is in $\mathbb{K}$, thus $\mathcal{A} \in \mathbf{H}\left(\mathbb{K}_{\text {fin }}\right)$.

Lemma 3.16. Let $\mathcal{A}$ be a WNM-chain. Then $\operatorname{ISP}_{U}(\mathcal{A})_{\text {fin }}=\mathbf{I S}(\mathcal{A})_{\text {fin }}$. 

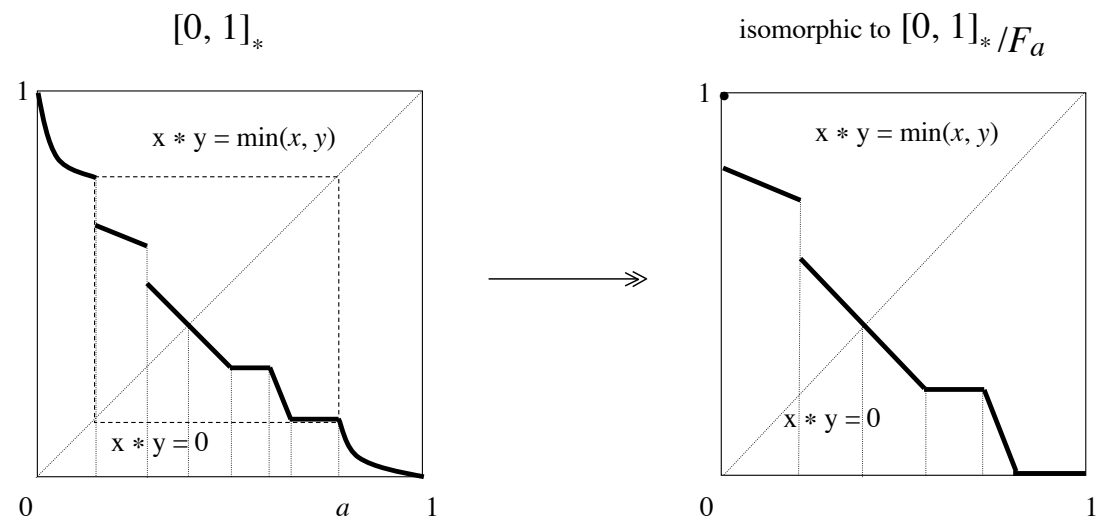

Figure 4: A WNM-t-norm satisfying the FPP such that $I_{1}=\{1\}$ (left) and its corresponding t-norm on the quotient algebra $[0,1]_{*} / F_{a}$ (right).

Proof. One direction is obvious. Due to the local finiteness of $\mathbb{W} \mathbb{N M}$, to prove the other one is equivalent to prove that $\operatorname{ISP}_{U}(\mathcal{A})$ is partially embeddable into $\mathbf{I S}(\mathcal{A})_{\text {fin }}$, which is equivalent by Proposition 2.12 to $\operatorname{ISP}_{U}(\mathcal{A}) \subseteq \mathbf{I S P}_{U}\left(\mathbf{I S}(\mathcal{A})_{\text {fin }}\right)$. Finally, the last inclusion is true because $\mathcal{A} \in \mathbf{I S P}_{U}\left(\mathbf{I S}(\mathcal{A})_{\text {fin }}\right.$ ) (recall that a first-order structure is embeddable into an ultraproduct of its finitely generated substructures, and in $\mathbb{W} \mathbb{N M}$ finitely generated algebras are finite).

Proposition 3.17. Let $\mathcal{A}$ be a WNM-chain. Then $\mathbf{H S P}_{U}(\mathcal{A})_{\text {fin }}=\mathbf{I S}(\mathcal{A})_{\text {fin }} \cup \mathbf{I S}\left(\left\{\mathcal{A} / F^{a}\right.\right.$ : $a \in N(\mathcal{A}) \cap A_{+}$and $\left.\left.I_{a} \neq\{a\}\right\}\right)_{\text {fin }}$.

Proof. $\mathbf{H S P}_{U}(\mathcal{A})_{\text {fin }}=\mathbf{H}\left(\mathbf{I S P}_{U}(\mathcal{A})\right)_{\text {fin }}=\left[\right.$ by Lemma 3.15] $\mathbf{H}\left(\operatorname{ISP}_{U}(\mathcal{A})_{f i n}\right)=[$ by Lemma 3.16] $\mathbf{H}\left(\mathbf{I S}(\mathcal{A})_{f i n}\right)=\mathbf{H}(\mathbf{I S}(\mathcal{A}))_{f i n}=\mathbf{H S}(\mathcal{A})_{f i n}=\mathbf{S H}(\mathcal{A})_{f i n}=\mathbf{S I S}\left(\left\{\mathcal{A} / F^{a}: a=\overline{1}^{\mathcal{A}}\right.\right.$ or $a \in$ $N(\mathcal{A}) \cap A_{+}$and $\left.\left.I_{a} \neq\{a\}\right\}\right)_{\text {fin }}=\mathbf{I S}\left(\left\{\mathcal{A} / F^{a}: a=\overline{1}^{\mathcal{A}} \text { or } a \in N(\mathcal{A}) \cap A_{+} \text {and } I_{a} \neq\{a\}\right\}\right)_{\text {fin }}$.

Corollary 3.18. Let $\mathcal{A}$ be a WNM-chain such that it has the maximum positive involutive element a with $I_{a} \neq\{a\}$, and for any other $b \in N(\mathcal{A}) \cap A_{+}, I_{b}$ is order-embeddable into $I_{a}$. Then, $\mathbf{H S P}_{U}(\mathcal{A})_{\text {fin }}=\mathbf{I S}(\mathcal{A})_{\text {fin }} \cup \mathbf{I S}\left(\mathcal{A} / F^{a}\right)_{\text {fin }}$.

The description of the classes $\mathbf{H S P}_{U}\left({ }_{-}\right)_{\text {fin }}$ leads to the following criterion to compare varieties generated by a finite family of chains.

Theorem 3.19. Let $n, m \geq 1$ be natural numbers and let $\mathcal{A}_{1}, \ldots, \mathcal{A}_{n}$ and $\mathcal{B}_{1}, \ldots, \mathcal{B}_{m}$ be WNM-chains such that for every $i$ there exists $a_{i} \in A_{i}$ and $b_{i} \in B_{i}$, positive involutive elements satisfying the conditions of the previous corollary. The following are equivalent:

(i) $\mathbf{V}\left(\mathcal{A}_{1}, \ldots, \mathcal{A}_{n}\right) \subseteq \mathbf{V}\left(\mathcal{B}_{1}, \ldots, \mathcal{B}_{m}\right)$

(ii) $\mathbf{I S}\left(\mathcal{A}_{1}, \ldots, \mathcal{A}_{n}, \mathcal{A}_{1} / F^{a_{1}}, \ldots, \mathcal{A}_{n} / F^{a_{n}}\right)_{\text {fin }} \subseteq \mathbf{I S}\left(\mathcal{B}_{1}, \ldots, \mathcal{B}_{m}, \mathcal{B}_{1} / F^{b_{1}}\right.$, $\left.\ldots, \mathcal{B}_{m} / F^{b_{m}}\right)_{\text {fin }}$.

(iii) 1. For every $i \in\{1, \ldots, n\}$, there is $j \in\{1, \ldots, m\}$ such that $\mathbf{I S}\left(\mathcal{A}_{i}\right)_{\text {fin }} \subseteq \mathbf{I S}\left(\mathcal{B}_{j}\right)_{\text {fin }}$ or $\mathbf{I S}\left(\mathcal{A}_{i}\right)_{\text {fin }} \subseteq \mathbf{I S}\left(\mathcal{B}_{j} / F^{b_{j}}\right)_{\text {fin }}$, and

2. for every $i \in\{1, \ldots, n\}$, there is $k \in\{1, \ldots, m\}$ such that $\mathbf{I S}\left(\mathcal{A}_{i} / F^{a_{i}}\right)_{\text {fin }} \subseteq$ $\mathbf{I S}\left(\mathcal{B}_{k}\right)_{f i n}$ or $\mathbf{I S}\left(\mathcal{A}_{i} / F^{a_{i}}\right)_{f i n} \subseteq \mathbf{I S}\left(\mathcal{B}_{k} / F^{b_{k}}\right)_{f i n}$. 
Proof. First observe that:

$\mathbf{V}\left(\mathcal{A}_{1}, \ldots, \mathcal{A}_{n}\right) \subseteq \mathbf{V}\left(\mathcal{B}_{1}, \ldots, \mathcal{B}_{m}\right)$ if, and only if, $\mathbf{V}\left(\mathcal{A}_{1}, \ldots, \mathcal{A}_{n}\right)_{F S I} \subseteq \mathbf{V}\left(\mathcal{B}_{1}, \ldots, \mathcal{B}_{m}\right)_{F S I}$.

By Jónsson's Lemma and being $\mathbb{W N M}$ locally finite, this is equivalent to:

$$
\mathbf{H S P}_{U}\left(\mathcal{A}_{1}, \ldots, \mathcal{A}_{n}\right)_{\text {fin }} \subseteq \mathbf{H S P}_{U}\left(\mathcal{B}_{1}, \ldots, \mathcal{B}_{m}\right)_{\text {fin }}
$$

By the previous corollary, this is equivalent to:

$$
\mathbf{I S}\left(\mathcal{A}_{1}, \ldots, \mathcal{A}_{n}, \mathcal{A}_{1} / F^{a_{1}}, \ldots, \mathcal{A}_{n} / F^{a_{n}}\right)_{\text {fin }} \subseteq \mathbf{I S}\left(\mathcal{B}_{1}, \ldots, \mathcal{B}_{m}, \mathcal{B}_{1} / F^{b_{1}}, \ldots, \mathcal{B}_{m} / F^{b_{m}}\right)_{\text {fin }}
$$

Therefore, we have proved $(i) \Leftrightarrow(i i)$.

$($ iii $) \Rightarrow($ ii $)$ is trivial.

(ii) $\Rightarrow$ (iii): Suppose that (iii) does not hold. Then, for instance, there exists $i \in$ $\{1, \ldots, n\}$ such that for every $j \in\{1, \ldots, m\}, \mathbf{I S}\left(\mathcal{A}_{i}\right)_{\text {fin }} \nsubseteq \mathbb{I S}\left(\mathcal{B}_{j}\right)_{\text {fin }}$ and $\mathbf{I S}\left(\mathcal{A}_{i}\right)_{\text {fin }} \nsubseteq$ $\mathbf{I S}\left(\mathcal{B}_{j} / F^{b_{j}}\right)_{\text {fin }}$. Therefore, there exist $\mathcal{C}_{1}, \ldots, \mathcal{C}_{m}, \mathcal{D}_{1}, \ldots, \mathcal{D}_{m} \in \mathbf{I S}\left(\mathcal{A}_{i}\right)_{\text {fin }}$ such that for every $j, \mathcal{C}_{j}$ is not embeddable into $\mathcal{B}_{j}$ and $\mathcal{D}_{j}$ is not embeddable into $\mathcal{B}_{j} / F^{b_{j}}$. Consider the subalgebra $\mathcal{C} \subseteq \mathcal{A}_{i}$ generated by $C_{1} \cup \ldots \cup C_{m} \cup D_{1} \cup \ldots \cup D_{m}$. Then, $\mathcal{C}$ is finite and it cannot belong to $\mathbf{I S}\left(\mathcal{B}_{1}, \ldots, \mathcal{B}_{m}, \mathcal{B}_{1} / F^{b_{1}}, \ldots, \mathcal{B}_{m} / F^{b_{m}}\right)_{f i n}$, so (ii) does not hold.

We know by the local finiteness that every subvariety of $\mathbb{W} \mathbb{N M}$ is generated by its finite chains; moreover, for every WNM-chain $\mathcal{A}$ we have described the class $\mathbf{H S P}_{U}(\mathcal{A})_{\text {fin }}$ which is exactly the class of finite chains in $\mathbf{V}(\mathcal{A})$; and finally the previous theorem shows how to compare varieties by using the finite subalgebras of their generators. Therefore, finite WNM-chains will play a central role in the task of classifying varieties of WNM-algebras.

Given a WNM-chain $\mathcal{A}$, the negation in $\mathcal{A}$ only depends on the negation in $\mathcal{N}(\mathcal{A})$, due to the properties of Lemma 3.2. Therefore, every WNM-chain is characterized by the NMsubalgebra defined by its involutive elements and by the number of non-involutive elements in their associated intervals. As in the case of NM-chains, some canonical representatives could be defined for the finite chains. Given $n \geq 1, l_{1}, \ldots, l_{n} \geq 0, \mathcal{A}_{l_{1}, \ldots, l_{n}}^{n}$ will denote the WNM-chain that has $n$ involutive elements and $l_{i}$ non-involutive elements in the constant interval of the $(i+1)$-th involutive element. It is clear that these chains generate pairwise different varieties. We can see an example in Figure 5.

\subsection{Generic WNM-chains}

In this section we will study the WNM-chains that generate the variety $\mathbb{W N M}$, i.e. the generic chains.

Definition 3.20. Let $\mathcal{A}$ be a WNM-chain. $\mathcal{A}$ is generic if, and only if, $\mathbf{V}(\mathcal{A})=\mathbb{W} \mathbb{N}$.

They can be characterized by using Theorem 2.8 and Proposition 3.17 in the following way.

Theorem 3.21. Let $\mathcal{A}$ be a WNM-chain. The following are equivalent:

(1) $\mathcal{A}$ is generic.

(2) For every $\varphi \in F m_{\mathcal{L}}, \mathcal{A}=\varphi \approx \overline{1}$ if, and only if, $\vdash_{\mathrm{WNM}} \varphi$.

(3) For every finite WNM-chain $\mathcal{C}$, either $\mathcal{C}$ is embeddable into $\mathcal{A}$ or there is a $\in N(\mathcal{A}) \cap A_{+}$ such that $I_{a} \neq\{a\}$ and $\mathcal{C}$ is embeddable into $\mathcal{A} / F^{a}$.

Some chains satisfy a condition stronger than (3), namely all finite chains are embeddable into them. This situation is characterized in the next proposition. 


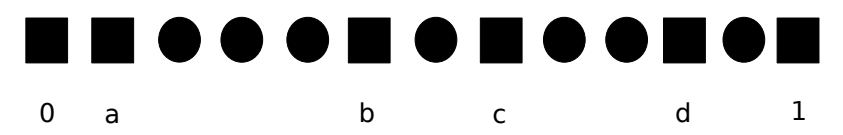

Figure 5: Example of a canonical finite WNM-chain, $\mathcal{A}_{0,3,1,2,1}^{6}$. Squares represent involutive elements, while circles represent the non-involutive ones. $b, c, d$ and 1 have some associated non-involutive elements, while $a$ (and, of course, 0) has a trivial associated interval.

Proposition 3.22. Let $\mathcal{A}$ be a WNM-chain. Then, all finite WNM-chains are embeddable into $\mathcal{A}$ if, and only if, $\mathcal{A}$ satisfies the following conditions:

1. The set $I_{\overline{1}} \mathcal{A}$ is infinite,

2. $\mathcal{A}$ has a negation fixpoint $f$ such that the set $I_{f}$ is infinite, and

3. Either there is an increasing sequence $\left\langle a_{n}: n \in \omega\right\rangle$ of involutive elements in $A_{-}$such that for every $n, k \geq 1$ there is $m \geq n$ such that the sets $I_{a_{m}}$ and $I_{\neg a_{m}}$ have both more than $k$ elements,

or there is an increasing sequence $\left\langle a_{n}: n \in \omega\right\rangle$ of involutive elements in $A_{+}$such that for every $n, k \geq 1$ there is $m \geq n$ such that the sets $I_{a_{m}}$ and $I_{\neg a_{m}}$ have both more than $k$ elements.

Proof. If $\mathcal{A}$ satisfies the conditions, it is obvious that every finite WNM-chain is embeddable into $\mathcal{A}$. In order to prove that the conditions are also necessary suppose that $\mathcal{A}$ satisfies the first and the second condition but not the third (if the first or the second condition fail, then it is easy to produce a finite chain that it is not embeddable into $\mathcal{A}$ ). Consider the set $X=\left\{a \in A_{-}: a\right.$ is involutive and $\left.\left|I_{a}\right|,\left|I_{\neg a}\right| \geq \omega\right\}$. This set must be finite (otherwise $\mathcal{A}$ would satisfy the third condition); suppose that $X$ has $m$ elements. For each involutive element $a \in A_{-}$, we define $r(a):=\min \left\{\left|I_{a}\right|,\left|I_{\neg a}\right|\right\}$. If $\left\{r(a): a \in A_{-} \backslash X, a=\neg \neg a\right\}$ is unbounded, we produce a sequence by choosing $a_{k} \in\left\{a \in A_{-} \backslash X: a=\neg \neg a\right.$ and $\left.r(a)=k\right\}$ for every $k \in \omega$ such that $\left\{a \in A_{-} \backslash X: a=\neg \neg a\right.$ and $\left.r(a)=k\right\} \neq \emptyset$. But then we would have a sequence satisfying the third condition, contradicting our assumption. Hence, there is an upper bound $k$ of $\left\{r(a): a \in A_{-} \backslash X, a=\neg \neg a\right\}$. Then, it is clear that the finite chain $\mathcal{A}_{k+1, k+1, \ldots, k+1}^{2 m+4}$ is not embeddable into $\mathcal{A}$.

Figure 6 shows an example of a generic WNM-chain defined by a WNM-t-norm satisfying this stronger condition. 


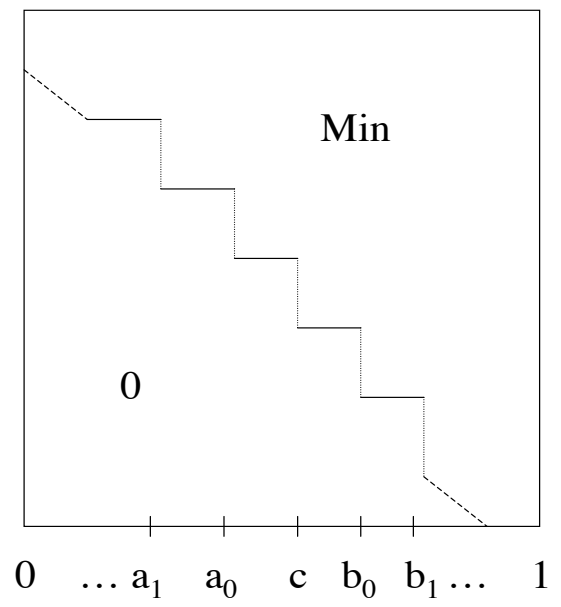

Figure 6: Example of a generic chain $\mathcal{A}$ defined by a WNM-t-norm over the real unit interval $[0,1]$. It has a decreasing sequence $\left\langle a_{n}: n \in \omega\right\rangle$ of involutive elements in the negative part such that $I_{a_{n}}=\left(a_{n+1}, a_{n}\right]$ for every $n$, an increasing sequence $\left\langle b_{n}=\neg a_{n}: n \in \omega\right\rangle$ of involutive elements in the positive part such that $I_{b_{n}}=\left(b_{n}, b_{n+1}\right]$ for every $n$, a fixpoint $c$ with $I_{c} \neq\{c\}$, and $I_{1} \neq\{1\}$.

Furthermore, we obtain the following characterization of generic standard WNM-chains.

Theorem 3.23. Let $\mathcal{A}$ be a standard WNM-chain. Then, $\mathcal{A}$ is generic if, and only if, it satisfies the following conditions:

1. $\mathcal{A}$ has a negation fixpoint $f$ such that the set $I_{f}$ is non-trivial, and

2. There is a sequence, either increasing or decreasing, $\left\langle a_{n}: n \in \omega\right\rangle$ of involutive elements in $A_{-}$such that for every $n \geq 0$ there is $m \geq n$ such that the sets $I_{a_{m}}$ and $I_{\neg a_{m}}$ are non-trivial.

Proof. Assume that $\mathcal{A}$ is generic. If there is a maximum constant interval $I_{a}$ (with possibly $a=1$ ), then every finite WNM-chain is embeddable into $\mathcal{A} / F^{a}$. Hence, by Proposition 3.22 , $\mathcal{A} / F^{a}$ satisfies the conditions, so also $\mathcal{A}$ satisfies them. Suppose now that the maximum constant interval does not exist. Since all finite chains are embeddable into $\mathcal{A} / F^{a}$ for some suitable $a$, it is clear that $\mathcal{A}$ has a negation fixpoint $f$ and the set $I_{f}$ is infinite. If it would not satisfy the other condition, then the set $\left\{a \in A_{-}: I_{a}\right.$ and $I_{\neg a}$ are infinite $\}$ would be finite, and then it would be possible to find a finite chain which we could not embed in any quotient of $\mathcal{A}$; a contradiction.

Conversely, suppose that $\mathcal{A}$ satisfies the two conditions. Then it is clear that every finite WNM-chain is embeddable into some quotient of $\mathcal{A}$. 


\subsection{T-norm based axiomatic extensions of the Weak Nilpotent Minimum logic and their standard completeness properties}

In this section we focus on varieties generated by t-norm-algebras, i.e. standard WNM-chains.

Lemma 3.24. Let $[0,1]_{*}$ be a standard WNM-chain. If $I_{1} \neq\{1\}$, then $\mathbf{H S P}_{U}\left([0,1]_{*}\right)_{\text {fin }}=$ $\mathbf{I S}\left([0,1]_{*}\right)_{\text {fin }}$.

Proof. Just apply the Corollary 3.18 with $a=1$.

This gives the following criterion to compare varieties generated by standard WNM-chains such that $I_{1} \neq\{1\}$.

Corollary 3.25. Let $\mathcal{A}$ and $\mathcal{B}$ be standard WNM-chains such that $I_{1}^{\mathcal{A}} \neq\{1\}$ and $I_{1}^{\mathcal{B}} \neq\{1\}$. Then the following are equivalent:

- $\mathbf{V}(\mathcal{A}) \subseteq \mathbf{V}(\mathcal{B})$

- $\mathbf{I S}(\mathcal{A})_{\text {fin }} \subseteq \mathbf{I S}(\mathcal{B})_{\text {fin }}$.

We can obtain similar results for t-norms satisfying the FPP.

Lemma 3.26. Let $[0,1]_{*}$ be a standard WNM-chain. If $* \in \mathbf{W N M - f i n ~ a n d ~} I_{a}$ is the maximum constant interval, then $\mathbf{H S P}_{U}\left([0,1]_{*}\right)_{\text {fin }}=\mathbf{I S}\left([0,1]_{*}\right)_{\text {fin }} \cup \mathbf{I S}\left([0,1]_{*} / F^{a}\right)_{\text {fin }}$.

Proof. By Corollary 3.18.

Corollary 3.27. Let $\mathcal{A}$ and $\mathcal{B}$ be standard WNM-chains with finite partition such that $I_{a}^{\mathcal{A}}$ and $I_{b}^{\mathcal{B}}$ are their maximum constants intervals respectively. Then the following are equivalent:

- $\mathbf{V}(\mathcal{A}) \subseteq \mathbf{V}(\mathcal{B})$

- $\mathbf{I S}(\mathcal{A})_{\text {fin }} \cup \mathbf{I S}\left(\mathcal{A} / F^{a}\right)_{\text {fin }} \subseteq \mathbf{I S}(\mathcal{B})_{\text {fin }} \cup \mathbf{I S}\left(\mathcal{B} / F^{b}\right)_{\text {fin }}$.

Notice that corollaries 3.25 and 3.27 give a classification of varieties generated by a standard WNM-chain (when the chains have $I_{1} \neq\{1\}$ or satisfy the FPP). Indeed, if $\mathcal{A}$ and $\mathcal{B}$ are standard WNM-chains under these conditions, the inclusion of the set of finite subalgebras of $\mathcal{A}$ into the set of finite subalgebras of $\mathcal{B}$ is easy to compute, since the possible finite subalgebras only depend on the partitions of $\mathcal{A}$ and $\mathcal{B}$. The results can be easily generalized to varieties generated by a family of standard WNM-chains.

Remark 3.28. It is easy to see that if $* \in \mathbf{W N M - f i n , ~ t h e n ~ a l l ~ t h e ~ c h a i n s ~ i n ~ t h e ~ v a r i e t y ~}$ $\mathbf{V}\left([0,1]_{*}\right)$ enjoy the FPP. Indeed, we can equationally express the maximum number of constant intervals that these chains can have in their partitions. Suppose, for instance, that $[0,1]_{*}$ is the standard WNM-chain depicted in Figure 2 and consider the following equations (recall the terms used in Proposition 3.4):

$$
\begin{aligned}
& \left(E_{1}\right) \neg \neg n(x) \rightarrow n(x) \approx \overline{1} \\
& \left(E_{2}\right)\left(\neg \neg x_{0} \leftrightarrow \neg x_{0}\right) \vee\left(\neg \neg x_{1} \leftrightarrow \neg x_{1}\right) \vee\left(\neg \neg x_{2} \leftrightarrow \neg x_{2}\right) \vee\left(\neg \neg p\left(x_{0}\right) \rightarrow p\left(x_{0}\right)\right) \vee\left(\neg \neg p\left(x_{1}\right) \rightarrow\right. \\
& \left.p\left(x_{1}\right)\right) \vee\left(\neg \neg p\left(x_{2}\right) \rightarrow p\left(x_{2}\right)\right) \vee\left(\neg \neg p\left(x_{0}\right) \rightarrow \neg \neg p\left(x_{1}\right)\right) \vee\left(\neg \neg p\left(x_{1}\right) \rightarrow \neg \neg p\left(x_{2}\right)\right) \approx \overline{1}
\end{aligned}
$$


It is not difficult to check that any WNM-chain satisfying $\left(E_{1}\right)$ has only involutive elements in the negative part, and any WNM-chain satisfying $\left(E_{2}\right)$ has at most 2 constant intervals in the positive part. Since these equations are valid in $[0,1]_{*}$, they are also valid in all the chains in $\mathbf{V}\left([0,1]_{*}\right)$, and hence all of them enjoy the FPP.

Given any standard WNM-chain $[0,1]_{*}$ it is obvious that the logic $\mathrm{L}_{*}$, i.e. the logic corresponding to the variety $\mathbf{V}\left([0,1]_{*}\right)$, enjoys the canonical $\mathcal{R} C$ with respect to $[0,1]_{*}$. Now we will study in which cases this standard completeness result can be improved. We start with t-norms satisfying the FPP.

Proposition 3.29. Let $* \in \mathbf{W N M - f i n , ~ l e t ~} I_{a}$ be its maximum constant interval (with possibly $a=1$ ) and let $\mathcal{A}$ be a countable $\mathrm{L}_{*}$-chain. Then:

- If $I_{\overline{1}}^{\mathcal{A}}=\left\{\overline{1}^{\mathcal{A}}\right\}$, then there exists an embedding from $\mathcal{A}$ into $[0,1]_{*}$.

- If $I_{\overline{1}_{\mathcal{A}}^{\mathcal{A}}}^{\mathcal{A}} \neq\left\{\overline{1}^{\mathcal{A}}\right\}$, then there exists an embedding from $\mathcal{A}$ into $[0,1]_{*} / F^{a}$.

Proof. We are assuming that $[0,1]_{*}$ has a finite partition. Let $r$ and $s$ be the number of intervals in the negative part, and respectively in the positive part, of $[0,1]_{*}$. Suppose that $I_{\overline{1} \mathcal{A}}^{\mathcal{A}}=\left\{\overline{1}^{\mathcal{A}}\right\}$. By the last remark we know that the number of intervals in the negative part (resp. in the positive part) of $\mathcal{A}$ is at most $r$ (resp. $s$ ). Take a finite WNM-subchain $\mathcal{B}$ satisfying the following construction rules:

1. Every unitary interval belonging to the partition of $\mathcal{A}$ is in $B$.

2. For every non-unitary constant interval of the partition of $\mathcal{A}$, one interior element of this interval and its upper bound belong to $B$.

3. For every involutive non-unitary interval in the negative part of the partition of $\mathcal{A}$, two different elements and their negations belong to $B$.

It is clear see that such a chain exists and it is a finite WNM-chain, subalgebra of $\mathcal{A}$, with the same number of intervals in the partition. By Lemma 3.26, there is an embedding $g: \mathcal{B} \hookrightarrow[0,1]_{*}$. Observe now that two different non-unitary intervals of the partition of $\mathcal{B}$ must be embedded into two different intervals of the partition of $[0,1]_{*}$ and also that as subalgebra two different intervals of the partition of $\mathcal{B}$ are contained in two different intervals of the partition of $\mathcal{A}$. Thus, remembering that the non-unitary intervals of $\mathcal{A}$ are countable and the ones in $[0,1]_{*}$ are continuous, and using that $\mathcal{A}$ and $\mathcal{B}$ have the same partition, we can define an embedding $f: \mathcal{A} \hookrightarrow[0,1]_{*}$. Assume now that $I_{\overline{1}}^{\mathcal{A}} \neq\left\{\overline{1}^{\mathcal{A}}\right\}$. This implies that $a$ is a positive element of $[0,1]_{*}$ (otherwise all positive elements in $[0,1]_{*}$ would be involutive and, since this property is equationally expressable by $\neg \neg p(x) \approx p(x)$ it would also be the case in $\mathcal{A}$ - a contriction with $I_{\overline{1}}^{\mathcal{A}} \neq\left\{\overline{1}^{\mathcal{A}}\right\}$ ). Therefore, $F^{a}$ is a proper filter and we can apply the previous reasoning to build an embedding from $\mathcal{A}$ into $[0,1]_{*} / F^{a}$.

Corollary 3.30. Let $* \in \mathbf{W N M - f i n ~ a n d ~ l e t ~} I_{a}$ be its maximum positive constant interval, if it exists. Then:

- If $a=1$ or $*$ has no positive constant intervals, then the logic $\mathrm{L}_{*}$ has the canonical SSC with respect to $[0,1]_{*}$. 
- If $a \neq 1$, then the logic $\mathrm{L}_{*}$ has the $S S C$ with respect to $\left\{[0,1]_{*},[0,1]_{*} / F^{a}\right\}$.

Now we turn to t-norms with an infinite partition.

Proposition 3.31. Given $* \in \mathbf{W N M} \backslash \mathbf{W N M - f i n , ~ a n ~} \mathrm{L}_{*}$-chain $\mathcal{C}$ and a finite partial subalgebra $\mathcal{B} \subseteq p$, we have:

- If $I_{1}^{*} \neq\{1\}$, then $\mathcal{B}$ is partially embeddable into $[0,1]_{*}$.

- If $I_{1}^{*}=\{1\}$, then $\mathcal{B}$ is partially embeddable into $[0,1]_{*}$ or there is a positive involutive element $a \in[0,1]$ with $I_{a}^{*} \neq\{a\}$ such that $\mathcal{B}$ is partially embeddable into $[0,1]_{*} / F^{a}$.

Proof. Since $\mathbb{W} \mathbb{N M}$ is locally finite, the subalgebra of $\mathcal{C}$ generated by $\mathcal{B}$ is also finite. Then, Proposition 3.17 gives the result.

Corollary 3.32. Given $* \in \mathbf{W N M} \backslash \mathbf{W N M - f i n , ~ w e ~ h a v e : ~}$

- If $I_{1}^{*} \neq\{1\}$, then the logic $\mathrm{L}_{*}$ has the canonical $\mathrm{FSRC}$ with respect to $[0,1]_{*}$.

- If $I_{1}^{*}=\{1\}$, then the logic $\mathrm{L}_{*}$ has the $\mathrm{FSRC}$ with respect to $\left\{[0,1]_{*}\right\} \cup\left\{[0,1]_{*} / F^{a}\right.$ : a is positive, involutive and $\left.I_{a} \neq\{a\}\right\}$.

Although in some cases the SRC holds for logics of WNM-t-norms with an infinite partition (for instance, when $[0,1]_{*}$ is a generic WNM-chain), it is false in general as the following examples show.

Example: Let $[0,1]_{*}$ be a standard WNM-chain with an infinite partition such that the number of positive constant intervals is finite, say $I_{a_{1}}^{*}, \ldots, I_{a_{n}}^{*}$. Assume that $I_{1}^{*} \neq\{1\}$. For every $i$, let $X_{i}$ be the set of these discontinuity points of the negation between $I_{a_{i}}^{*}$ and $I_{a_{i+1}}^{*}$, let $Y_{i 1}$ be the set of accumulation points of $X_{i}$ which are a limit of an increasing sequence of elements of $X_{i}$, and let $Y_{i 2}$ be the set of accumulation points of $X_{i}$ which are a limit of a decreasing sequence of elements of $X_{i}$. Take $a \in I_{1}^{*} \backslash\{1\}$ and let $\mathcal{A}$ be the countable subalgebra of $[0,1]_{*}$ generated by the rational numbers in $[0, a]$. It is clear that $\mathcal{A}$ is subdirectly irreducible. Assume that there is $i$ such that $X_{i}$ is infinite and $Y_{i 1}$ or $Y_{i 2}$ is finite. Then:

1. If $Y_{i 1}$ is finite, we can produce a new countable WNM-chain $\mathcal{B}$ by adding to $\mathcal{A}$ a new accumulation point to $Y_{i 1}$.

2. If $Y_{i 2}$ is finite, we can produce a new countable WNM-chain $\mathcal{B}$ by adding to $\mathcal{A}$ a new accumulation point to $Y_{i 2}$.

In both cases, $\mathcal{B} \in \mathbf{V}\left([0,1]_{*}\right)$, since every finite subalgebra of $\mathcal{B}$ is embeddable into $[0,1]_{*}$, but clearly $\mathcal{B}$ is not embeddable into $[0,1]_{*}$. Therefore, $\mathrm{L}_{*}$ has not the $\mathrm{S} \mathcal{R} \mathrm{C}$.

An analogous reasoning is possible when the number of negative constant intervals is finite.

Example: Let $[0,1]_{*}$ be a standard WNM-chain with an infinite partition such that 1 is the only accumulation point of positive constant intervals. Consider the formula $\varphi_{>}(x, y):=$ $(y \rightarrow x) \wedge((x \rightarrow y) \rightarrow y)$. The following claim is easy to check.

Claim: For every WNM-chain $\mathcal{A}$ and every $a, b \in A$ we have:

$\varphi_{>}^{\mathcal{A}}(a, b)=\overline{1}^{\mathcal{A}}$ iff $a>b$ or $a=b=\overline{1}^{\mathcal{A}}$.

Using this formula, we can formulate an infinite semantical derivation, $\left\{\varphi_{>}\left(\neg \neg p\left(x_{i+1}\right), \neg \neg p\left(x_{i}\right)\right)\right.$ : $i \geq 1\} \cup\left\{\varphi_{>}\left(\neg \neg p\left(x_{i}\right), p\left(x_{i}\right)\right): i \geq 1\right\} \cup\left\{\varphi_{>}\left(\neg \neg p\left(x_{0}\right), \neg \neg p\left(x_{i}\right)\right): i \geq 1\right\}=_{[0,1]_{*}} \neg \neg p\left(x_{0}\right) \vee$ 
$\neg \neg p\left(x_{1}\right)$, but it is not valid if we consider only a finite subset of the premisses, so $\mathrm{L}_{*}$ has not the $\mathrm{SRC}$.

An analogous reasoning is possible when the only accumulation point of positive constant intervals is the infimum of the positive elements.

\subsection{Axiomatization of some t-norm based extensions of the Weak Nilpotent Minimum logic}

In this section we give finite equational bases for some varieties generated by standard WNMchains, or equivalently finite axiomatizations for some t-norm based extensions of WNM. Since every variety is generated by its finite chains, the equational base essentially has to describe these finite chains. More precisely:

Lemma 3.33. Given a WNM-chain $\mathcal{A}$, the following statements are equivalent:

1. The variety $\mathbf{V}(\mathcal{A})$ is axiomatized by the equations $\Sigma \subseteq E q_{\mathcal{L}}$.

2. For every finite WNM-chain $\mathcal{C}, \mathcal{C} \in \mathbf{H S P}_{U}(\mathcal{A})$ iff $\mathcal{C}=\Sigma$.

3. For every finite WNM-chain $\mathcal{C}, \mathcal{C} \in \mathbf{I S}(\mathcal{A}) \cup \mathbf{I S}\left(\left\{\mathcal{A} / F^{a}: a \in N(\mathcal{A}) \cap A_{+}\right.\right.$and $\left.\left.I_{a} \neq\{a\}\right\}\right)$ iff $\mathcal{C} \models \Sigma$.

We will focus on the last condition, which is the most descriptive.

First, we consider some easy observations on the equations in the language of WNM.

Lemma 3.34. Let $\mathcal{A}$ be a WNM-algebra, let $\varphi \approx \psi \in E q_{\mathcal{L}}$ be an equation and $\Sigma=\left\{\varphi_{i} \approx\right.$ $\left.\psi_{i}: i<n\right\} \subseteq E q_{\mathcal{L}}$ be a finite set of equations. Then:

1. $\mathcal{A}=\varphi \approx \psi$ if, and only if, $\mathcal{A} \models \varphi \leftrightarrow \psi \approx \overline{1}$, and

2. $\mathcal{A}=\Sigma$ if, and only if, $\mathcal{A} \models\left(\varphi_{0} \leftrightarrow \psi_{0}\right) \& \ldots \&\left(\varphi_{n-1} \leftrightarrow \psi_{n-1}\right) \approx \overline{1}$.

Therefore, every finite equational base can be reduced to one single equation whose second member is the constant for the neutral element of the monoid. Using this and the following lemma we can produce an equational base for the variety generated by a finite family of WNM-chains, whenever we have an equational base for the variety generated by each chain of the family.

Lemma 3.35. Let $t_{0}\left(\overline{x_{0}}\right), \ldots, t_{n}\left(\overline{x_{n}}\right) \in F m_{\mathcal{L}}$, where $\overline{x_{0}}, \ldots, \overline{x_{n}}$ denote pairwise disjoint sets of variables. Let $\mathcal{A}$ be a WNM-chain. Then, $\mathcal{A} \models t_{0}\left(\overline{x_{0}}\right) \vee \ldots \vee t_{n}\left(\overline{x_{n}}\right) \approx \overline{1}$ if, and only if, there exists $i \leq n$ such that $\mathcal{A}=t_{i}\left(\overline{x_{i}}\right) \approx \overline{1}$.

Proposition 3.36. Let $\left\{\mathcal{C}_{1}, \ldots, \mathcal{C}_{n}\right\}$ be a finite set of WNM-chains. Suppose that for each $i \in$ $\{1, \ldots, n\}, t_{i} \approx \overline{1}$ is an equation axiomatizing $\mathbf{V}\left(\mathcal{C}_{i}\right)$, in such a way that the sets of variables of these equations are pairwise disjoint. Then, the equation $t_{1} \vee \ldots \vee t_{n} \approx \overline{1}$ axiomatizes the variety $\mathbf{V}\left(\left\{\mathcal{C}_{1}, \ldots, \mathcal{C}_{n}\right\}\right)$.

Proof. It follows from the last results and the fact that $\mathbf{H S P}_{U}\left(\mathcal{C}_{1}, \ldots, \mathcal{C}_{n}\right)=\mathbf{H S P}_{U}\left(\mathcal{C}_{1}\right) \cup$ $\ldots \cup \mathbf{H S P}_{U}\left(\mathcal{C}_{n}\right)$. 
In the following we provide some examples of t-norm based axiomatic extensions of WNM for which we are able to give efectively a finite axiomatization.

Examples: Let $*$ be a WNM-t-norm, let $[0,1]_{*}$ be its corresponding standard WNMalgebra and let $\mathrm{L}_{*}$ be the axiomatic extension of WNM corresponding to the variety $\mathbf{V}\left([0,1]_{*}\right)$. Our aim is to find a set of axiom schemata such that, added to the Hilbert-style system for WNM, give a calculus for $\mathrm{L}_{*}$ (or equivalently, to find a set of equations such that, added to the equational base for $\mathbb{W} \mathbb{N M}$, give an equational base for $\left.\mathbf{V}\left([0,1]_{*}\right)\right)$. We will use the terms introduced in Proposition 3.4.

1. If $[0,1]_{*}$ is a generic WNM-t-norm (i.e. it satisfies the conditions of Theorem 3.23), then $\mathrm{L}_{*}$ is just WNM, and hence there is no need for additional axioms.

2. Suppose that $[0,1]_{*}$ satisfies the following conditions:

- The partition of $[0,1]_{*}$ has no constant interval in the negative part.

- $[0,1]_{*}$ has a negation fixpoint.

- The partition of $[0,1]_{*}$ has infinitely many constant intervals in the positive part (i.e. $* \in \mathbf{W N M} \backslash \mathbf{W N M - f i n )}$.

It is clear that for every finite WNM-chain $\mathcal{C}, \mathcal{C} \in \mathbf{I S}\left([0,1]_{*}\right) \cup \mathbf{I S}\left(\left\{[0,1]_{*} / F^{a}: a \in\right.\right.$ $N\left([0,1]_{*}\right) \cap\left([0,1]_{*}\right)_{+}$and $\left.\left.I_{a} \neq\{a\}\right\}\right)$ iff all the negative elements in $\mathcal{C}$ are involutive. Therefore, the variety generated by $[0,1]_{*}$ is axiomatized by the following equation: ${ }^{8}$

$$
\neg \neg n(x) \approx n(x)
$$

Notice that the symmetric situation (no constant intervals in the positive part, while infinitely many in the negative part) is axiomatized by:

$$
\neg \neg p(x) \approx p(x)
$$

Of course, the two equations together would give the variety generated by $[0,1]_{\mathrm{NM}}$, which can be axiomatized just by:

$$
\neg \neg x \approx x
$$

3. Suppose that $[0,1]_{*}$ satisfies the following condition:

- There is a sequence, either increasing or decreasing, $\left\langle a_{n}: n \in \omega\right\rangle$ of involutive elements in $A_{-}$such that for every $n \geq 0$ there is $m \geq n$ such that the sets $I_{a_{m}}$ and $I_{\neg a_{m}}$ are non-trivial.

i.e. just the second condition required for generic standard WNM-chains in Theorem 3.23. On the one hand, it is clear that for every finite WNM-chain $\mathcal{C}, \mathcal{C} \in \mathbf{I S}\left([0,1]_{*}\right) \cup$ $\mathbf{I S}\left(\left\{[0,1]_{*} / F^{a}: a \in N[0,1]_{*}\right) \cap\left([0,1]_{*}\right)_{+}\right.$and $\left.\left.I_{a} \neq\{a\}\right\}\right)$ iff $\mathcal{C}$ has no negation fixpoint. On the other hand, in the papers $[22,23]$, while studying the varieties generated by

\footnotetext{
${ }^{8}$ Its associated logic has been already studied in [13] under with a different name, MTL $\left[D_{\wedge}\right]$, and a different axiomatization. In particular, the authors proved the $\mathrm{S} \mathcal{R} \mathrm{C}$ for this logic.
} 
perfect IMTL-algebras and perfect MTL-algebras, the authors proved that a WNMchain is perfect iff it has no negation fixpoint. Therefore, the equation for perfect MTLchains will be enough to obtain an equational base for the variety we are considering now:

$$
\left(\neg(\neg x)^{2}\right)^{2} \approx \neg\left(\neg x^{2}\right)^{2}
$$

4. Take $* \in \mathbf{W N M - f i n ~ s u c h ~ t h a t ~ t h e ~ p a r t i t i o n ~ o f ~}[0,1]_{*}$ has no involutive intervals. Let $r$ and $s$ be respectively the number of constant intervals in the negative and in the positive part. Then, due to the symmetry properties of negation functions, we obtain that:

- If $[0,1]_{*}$ has no negation fixpoint, then $s=r+1$.

- If $[0,1]_{*}$ has negation fixpoint, then $s=r$.

Observe that these chains (we can see two examples in Figure 7) have a finite number of involutive elements: 0 and the right extreme of each constant interval:

- If $[0,1]_{*}$ has no negation fixpoint, then it has $2 r+2$ involutive elements.

- If $[0,1]_{*}$ has negation fixpoint, then it has $2 r+1$ involutive elements.
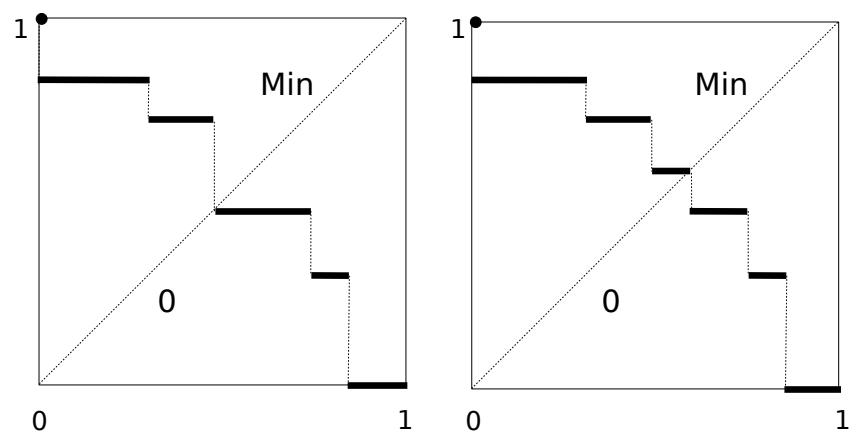

Figure 7: Two examples of standard WNM-chains satisfying the FPP with no involutive intervals. The chain on the left hand side has no negation point, while the chain on the right hand side has it.

Therefore, in order to axiomatize this kind of varieties we only need an equation giving an upper bound to the number of involutive elements. It is easy to check that a WNMchain $\mathcal{A}$ has at most $k$ involutive elements if, and only if, the following equation is valid in $\mathcal{A}$ :

$$
\bigvee_{i<k}\left(\neg x_{i} \rightarrow \neg x_{i+1}\right) \approx \overline{1}
$$


For instance, to axiomatize the varieties corresponding to the chains in Figure 7 , we would take the equation with $k=6$ (for the chain on the left hand side) and the equation with $k=7$ (for the chain on the right hand side).

5. Finally, assume that $* \in \mathbf{W N M - f i n}$ and the partition of $[0,1]_{*}$ has some involutive intervals. We have not found an equational base for every WNM-t-norm under these conditions. However, we will illustrate with some example how it could be done when the partition has a small number of intervals. For instance, suppose that $*$ is the t-norm depicted in Figure 2. In this case the equational base only needs to force the chains to have no constant intervals in the negative part and at most 2 in the positive part. Thus we take the equations:

$$
\neg \neg n(x) \approx n(x)
$$

and

$$
\bigvee_{i<3}\left(\neg x_{i} \leftrightarrow \neg \neg x_{i}\right) \vee \bigvee_{i<3}\left(\neg \neg p\left(x_{i}\right) \rightarrow p\left(x_{i}\right)\right) \vee \bigvee_{i<2}\left(\neg \neg p\left(x_{i}\right) \rightarrow \neg \neg p\left(x_{i+1}\right)\right) \approx \overline{1}
$$

Consider now the chain in Figure 8 where some more restrictions must be described in the equations.

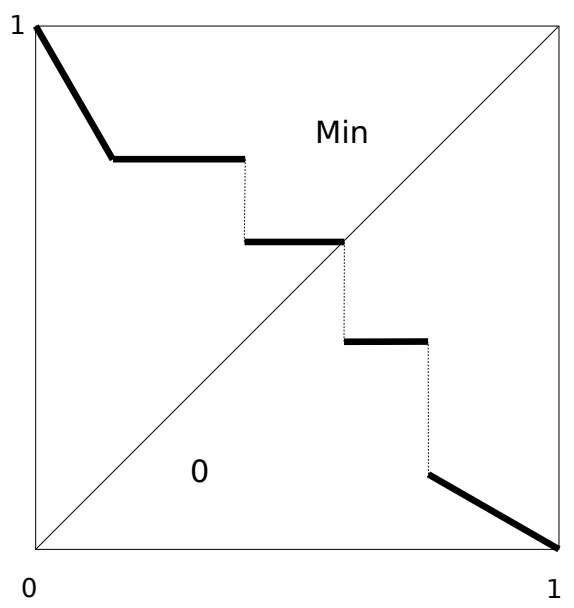

Figure 8: An example of a standard WNM-chain satisfying the FPP with involutive intervals.

In this case we take the following equational base:

$\bigvee_{i<3}\left(\neg \neg n\left(x_{i}\right) \rightarrow n\left(x_{i}\right)\right) \vee \bigvee_{i<2}\left(\neg \neg n\left(x_{i}\right) \rightarrow \neg \neg n\left(x_{i+1}\right)\right) \approx \overline{1}$

(there are at most two constant intervals in the negative part)

$\bigvee_{i<2}\left(\neg x_{i} \leftrightarrow \neg \neg x_{i}\right) \vee \bigvee_{i<2}\left(\neg \neg p\left(x_{i}\right) \rightarrow p\left(x_{i}\right)\right) \vee\left(\neg \neg p\left(x_{0}\right) \rightarrow \neg \neg p\left(x_{1}\right)\right) \approx \overline{1}$

(there is at most one constant interval in the positive part)

$\bigvee_{i<2}\left(\neg \neg n\left(x_{i}\right) \rightarrow n\left(x_{i}\right)\right) \vee \bigvee_{i<2}\left(\neg \neg n\left(x_{i}\right) \rightarrow \neg \neg n\left(x_{i+1}\right)\right) \vee\left(\neg \neg n(y) \rightarrow \neg \neg n\left(x_{1}\right)\right) \vee$ $\left(\neg \neg n\left(x_{0}\right) \rightarrow \neg \neg n(y)\right) \approx \overline{1}$ 
(if there are two constant intervals in the negative part, then there are no involutive elements between them)

$$
\bigvee_{i<2}\left(\neg \neg n\left(x_{i}\right) \rightarrow n\left(x_{i}\right)\right) \vee \bigvee_{i<2}\left(\neg \neg n\left(x_{i}\right) \rightarrow \neg \neg n\left(x_{i+1}\right)\right) \vee\left(\neg \neg n\left(x_{0}\right) \leftrightarrow \neg n\left(x_{0}\right)\right) \approx \overline{1}
$$

(if there are two constant intervals in the negative part, then the right extreme of the second one is the negation fixpoint)

$\left(\neg \neg n\left(x_{0}\right) \rightarrow n\left(x_{0}\right)\right) \vee\left(\neg \neg n\left(y_{0}\right) \rightarrow \neg \neg n\left(y_{1}\right)\right) \vee\left(\neg \neg n\left(y_{1}\right) \rightarrow \neg \neg n\left(x_{0}\right)\right) \approx \overline{1}$

(if there is a constant interval in the negative part, then there is at most one negative involutive element above it)

$\left(\neg x_{0} \leftrightarrow \neg \neg x_{0}\right) \vee\left(\neg \neg p\left(x_{0}\right) \rightarrow p\left(x_{0}\right)\right) \vee\left(\neg y_{0} \leftrightarrow \neg \neg y_{0}\right) \vee\left(\neg \neg p\left(x_{0}\right) \rightarrow \neg \neg p\left(y_{0}\right)\right) \approx \overline{1}$

(if there is a constant interval in the positive part, then there are no positive involutive elements below it)

\section{Conclusions}

In this paper we have studied a particular variety of MTL-algebras, $\mathbb{W} \mathbb{N}$. After presenting the description and axiomatization of the varieties formed by the involutive members obtained in [14], we have achieved the following new results:

- $\mathbb{W N M}$ is a locally finite variety, so it has the FEP and the FMP and the corresponding logic is decidable. Obviously, these properties are inherited by all the subvarieties and axiomatic extensions, respectively.

- We have studied WNM-t-norms. In particular, we have characterized the generic tnorms, we have given criteria to compare their generated varieties and we have studied their standard completeness properties.

- We have given equational bases for some varieties generated by a finite family of standard WNM-chains.

As a matter of future research it would be interesting to find a general method to axiomatize t-norm based extensions of WNM, or even all axiomatic extensions of WNM.

Acknowledgements: The authors acknowledge partial support of the Spanish projects MULOG TIN2004-07933-C03-01/02 and MTM2004-031012001-3329, and the Catalan project 2005SGR00083. We are indebted with professor Josep Pla, whose useful comments helped us in the proof of Proposition 3.22, and with Rostislav Horčík for his reading of the paper and his valuable remarks. Finally, we want to thank the anonymous referee for his comments and corrections that helped us in improving the submitted version of the paper.

\section{References}

[1] W. J. Blok And D. Pigozzi. On the structure of varieties with equationally definable principal congruences I, Algebra Universalis 15 (1982) 195-227.

[2] W. J. Blok And D. Pigozzi. Algebraizable logics, Memoirs of the American Mathematical Society 396, vol 77, 1989. 
[3] W. J. Blok And D. Pigozzi. Abstract Algebraic Logic and the Deduction Theorem, To appear in Bulletin of Symbolic Logic.

[4] W. J. Blok and C. J. Van Alten. The finite embeddability property for residuated lattices, pocrims and BCK-algebras, Algebra Universalis 48 (2002) 253-271.

[5] S. Burris and H. P. Sankappanavar. A course in Universal Algebra, Springer Verlag, New York, 1981.

[6] A. Ciabattoni, F. Esteva and L. Godo. T-norm based logics with $n$-contraction, Neural Network World 5 (2002) 441-452.

[7] P. Cintula, F. Esteva, J. Gispert, L. Godo, F. Montagna and C. Noguera. Distinguished algebraic semantics for t-norm based fuzzy logics: methods and algebraic equivalencies, Submitted, 2007.

[8] M. Dummett. A propositional calculus with denumerable matrix, The Journal of Symbolic Logic 24 (1959) 97-106.

[9] F. Esteva and X. Domingo. Sobre negaciones fuertes y débiles en [0,1], Stochastica 4 (1980) 141-166.

[10] F. Esteva And L. Godo. Monoidal t-norm based Logic: Towards a logic for leftcontinuous t-norms, Fuzzy Sets and Systems 124 (2001) 271-288.

[11] F. Esteva, L. Godo, And C. Noguera. On expansions of t-norm based logics with truth-constants, To appear in the book Fuzzy Logics and Related Structures (S. Gottwald, P. Hájek, U. Höhle and E.P. Klement eds.), 2007

[12] J. Fodor. Nilpotent minimum and related connectives for fuzzy logic, Proc. of FUZZIEEE'95, 1995, pp. 2077-2082.

[13] A. García-Cerdaña, C. Noguera and F. Esteva. On the scope of some formulas defining additive connectives in fuzzy logics, Fuzzy Sets and Systems 154 (2005) 56-75.

[14] J. Gispert. Axiomatic extensions of the nilpotent minimum logic, Reports on Mathematical Logic 37 (2003) 113-123.

[15] K. GöDEL. Zum intuitionistischen Aussagenkalkl, Anzeiger Akademie der Wissenschaften Wien, Math. naturwiss. Klasse 69 (1932), 65-66.

[16] S. Gotwwald. A Treatise on Many-Valued Logics, Studies in Logic and Computation, vol. 9, Research Studies Press Ltd., 2001.

[17] P. HÁJeK. Metamathematics of fuzzy logic, vol. 4 in Trends in Logic, Kluwer, Dordrecht, 1998.

[18] U. HöHLE. Commutative, residuated l-monoids, In Non-Classical Logics and Their Applications to Fuzzy Subsets (U. Höhle and E. P. Klement eds.), Kluwer Acad. Publ., Dordrecht (1995) 55-106.

[19] R. Horčík, C. Noguera, And M. Petrík. On n-contractive fuzzy logics, Mathematical Logic Quarterly 53 (2007) 268-288. 
[20] S. Jenei and F. Montagna. A proof of standard completeness for Esteva and Godo's logic MTL, Studia Logica 70 (2002) 183-192.

[21] C. NoguerA, Algebraic study of axiomatic extensions of triangular norm based fuzzy logics, Ph. D. dissertation, Universitat de Barcelona, 2006.

[22] C. Noguera, F. Esteva and J. Gispert. Perfect and bipartite IMTL-algebras and disconnected rotations of prelinear semihoops, Archive for Mathematical Logic 44 (2005) 869-886.

[23] C. Noguera, F. Esteva And J. Gispert. On some varieties of MTL-algebras, Logic Journal of the IGPL 13 (2005) 443-466.

[24] C. Noguera, F. Esteva and J. Gispert. On varieties generated by Weak Nilpotent Minimum t-norms, Proceedings of Fourth EUSFLAT, Barcelona, 2005, pp. 866-871.

[25] D. PEI. The equivalent forms of fuzzy logic systems NM and IMTL, Fuzzy Sets and Systems 138 (2003) 187-195.

[26] E. Trillas. Sobre funciones de negación en la teoría de conjuntos difusos, Stochastica 3 (1979) 47-60. English translation in Advances in Fuzzy logic (S. Barro, A. Bugarin and A. Sobrino eds.), Public. Univ. Santiago de Compostela (Spain) pp. 31-45. 